\title{
Regularization of energy-momentum tensor correlators and parity-odd terms
}

\author{
Loriano Bonora, ${ }^{a, b}$ Antônio Duarte Pereira ${ }^{a, c}$ and Bruno Lima de Souza ${ }^{a, b}$ \\ ${ }^{a}$ International School for Advanced Studies (SISSA), \\ Via Bonomea 265, 34136 Trieste, Italy \\ ${ }^{b}$ INFN, Sezione di Trieste, \\ Trieste, Italy \\ ${ }^{c}$ Universidade Federal Fluminense (UFF), Instituto de Física, \\ Campus da Praia Vermelha, Avenida General Milton Tavares de Souza s/n, \\ 24210-346, Niterói, RJ, Brasil \\ E-mail: bonora@sissa.it, aduarte@if.uff.br, blima@sissa.it
}

ABSTRACT: We discuss the problem of regularizing correlators in conformal field theories. The only way to do it in coordinate space is to interpret them as distributions. Unfortunately except for the simplest cases we do not have tabulated mathematical results. The way out we pursue here is to go to momentum space and use Feynman diagram techniques and their regularization methods. We focus on the energy-momentum tensor correlators and, to gain insight, we compute and regularize 2-point functions in $2 d$ with various techniques both in coordinate space and in momentum space, obtaining the same results. Then we do the same for 2-point functions in $4 d$. Finally we turn to 3 -point function in $4 d$, and concentrate on the parity-odd part. We derive in particular the regularized trace and divergence of the energy-momentum tensor in a chiral fermion model. We discuss the problems related to the parity-odd trace anomaly.

Keywords: Conformal and W Symmetry, Anomalies in Field and String Theories

ARXIV EPRINT: 1503.03326 


\section{Contents}

1 Introduction $\quad \mathbf{2}$

1.1 General formulas for the trace and divergence of the e.m. tensor 3

2 2-point function of e.m. tensors in $2 d$ and trace anomaly 5

2.1 Ambiguities 8

3 Parity-odd terms in $2 d r$

3.1 Using symmetries 8

$\begin{array}{ll}3.2 & \text { The embedding formalism }\end{array}$

$\begin{array}{lll}3.3 & \text { Differential regularization of the parity-odd part } & 10\end{array}$

3.4 Ambiguities in the parity-odd part 11

4 The Feynman diagrams method in $2 d \quad 12$

5 2-point correlator of e.m. tensors in $4 d \quad \mathbf{1 5}$

$\begin{array}{ll}5.1 \text { Differential regularization of the correlator } & 15\end{array}$

$\begin{array}{ll}5.2 \text { 2-point correlator with Feynman diagrams } & 16\end{array}$

6 The 3-point correlator $\quad 18$

$\begin{array}{lll}6.1 & \text { No-go for parity-odd contributions } & 19\end{array}$

6.2 The "bare" parity-odd 3-point correlator 20

6.3 Relevant Fourier transforms 22

6.4 The parity-odd 3-point correlator with Feynman diagrams 24

$\begin{array}{ll}\text { 6.4.1 The trace } & 24\end{array}$

$\begin{array}{lll}6.4 .2 & \text { The divergence } & 26\end{array}$

$\begin{array}{lll}6.4 .3 & \text { (Partial) conclusion } & 27\end{array}$

$\begin{array}{lll}7 & \text { The ugly duckling anomaly } & 28\end{array}$

8 Conclusions $\quad 30$

$\begin{array}{ll}\text { A Direct computation for a chiral fermion in } 2 d & 31\end{array}$

B The chiral fermion model in $4 d \quad 33$

C Regularization formulas in $2 d$ and $4 d \quad 34$

$\begin{array}{ll}\text { D Fourier transforms } & 35\end{array}$

$\begin{array}{ll}\text { E Conservation of the e.m. tensor } & 36\end{array}$ 


\section{Introduction}

In recent years CFT in $4 d$ has been receiving an increasing attention. The reason is wellknown, it is due in part to being one of the poles in the AdS/CFT correspondence, in part to the new applications to strongly correlated systems and in part to the increasing interest in applying the standard model of elementary particles to very high energy problems and its coupling to gravity. In turn this has spurred a lot of interest and activity in the theoretical aspects of conformal symmetry and conformal field theories. Recent reviews on the latter are [1, 2], older references relevant to the content of this paper are [3, 4]. One of the most striking recently obtained results is the derivation of the general structures of conformal covariant correlators and OPE's of any kind of tensor fields in coordinate space, [5-11]. The analysis of 3-point functions of conserved currents and the energy-momentum tensor was also considered in momentum space, [12-14].

The above mentioned correlators in coordinate space are in general unregulated expressions, in that they have singularities at coincident points. For convenience we call them bare. The natural way to regularize them is provided by distribution theory. This is clear in theory, in practice it is not so simple because, except for the simplest cases, one has to do with formidable expressions. In the coordinate representation a rather natural technique is provided by the so-called differential regularization, [15-17]. However this technique does not seem to be in general algorithmic (see below) and a good deal of guesswork is needed in order to obtain sensible expressions.

Regularizing correlators is not simply a procedure (legitimately) required by mathematics. Singularities in correlators usually contain useful information. For instance in correlators of currents or energy-momentum tensors singularities provide information about the coupling to gauge potentials and to gravity, respectively. This is the case of anomalies, which are a typical result of regularization processes, though independent of them. Regularized correlators are also necessary in the Callan-Symanzik equation, [18]. In summary, regularizing conformal correlators is the next necessary step after deriving their (unregulated or "bare") expressions.

As was said above, however, the process of regularizing higher order correlators in coordinate space representation with differential regularization does not seem to be algorithmic. For definiteness we concentrate here on the 2- and 3-point functions of the energy-momentum tensor. We show that we have a definite rule to regularize the 2-point correlators in coordinate space by means of differential regularization, but when we come to the 3-point function there is a discontinuity which does not allow us to extend the rule valid for the 2-point one. To understand the origin of the problem we resort to a model, the model of a free chiral fermion, in momentum representation. Using one-loop Feynman diagrams we can determine completely the 3-point correlator of the e.m. tensor and regularize it with standard dimensional regularization techniques. The idea is to Fourier anti-transform it in order to shed light on the regularization in the coordinate representation. For two reasons we concentrate on the parity-odd part, although the extension to the parity-even part is straightforward. The first reason is the presence of the Levi-Civita tensor which limits the number of terms to a more manageable amount, while preserving all the general features of the problem. 
The second reason is more important: the appearance of the Pontryagin density in the trace anomaly of this model. This parity-odd anomaly has been recalculated explicitly in [22] after the first appearance in [19, 20], with different methods. If one uses Feynman diagram techniques the basic evaluation is that of the triangle diagram. Now, it has been proved recently (this is one of the general results mentioned above) that the parity-odd part of the 3-point function of the energy-momentum tensor in the coordinate representation vanishes identically, $[7,8]$. Therefore it would seem that there is a contradiction with the existence of a parity-odd part in the trace of the e.m. tensor. Although this argument is rather naive and forgetful of the subtleties of quantum field theory, it seems to be widespread. Therefore we think it is worth clarifying it. We show below that in fact there is no contradiction: a vanishing parity-odd "bare" 3-point function of the energy-momentum tensor must in fact coexist with a nonvanishing parity-odd part of the trace anomaly.

The paper is organized as follows. In the next three sections we thoroughly analyse the $2 d$ case. The reason is that, although the results are known, in $2 d$ many problems that will appear in higher dimensions are already present and can be fully solved. So $2 d$ is a useful playground for the rest of the paper. In section 2 and 3 we consider the problem of regularizing the 2-point function of e.m. tensors in $2 d$ using the techniques of differential regularization (for the various techniques used, see [25-27]). In section 4 we analyze the 2-point function of the e.m. tensor using Feynman diagrams techniques. In section 5 we compute the 2-point function of e.m. tensors in $4 d$ both using differential regularization and Feynman diagrams. In section 6 we review a general no-go argument concerning parity-odd contributions in the 3-point function of e.m. tensors, we explicitly compute the parity-odd part of the correlator of three e.m. tensors in the chiral fermion model in $4 d$ in coordinate representation and show that it identically vanishes. We repeat the last computation using Feynman diagrams and regularize it, and show how it gives rise to the parity-odd trace anomaly. We show that irreducible Lorentz components of the correlators, in particular those containing the trace and the traceless part of the e.m. tensor, must be regularized separately. We also discuss the connection of the anomaly with the e.m. conservation. We show that in general regularization breaks covariance and counterterms must be subtracted in order to recover it. In section 7 we discuss the prejudices on the existence of the Pontryagin anomaly.

To complete this introduction we present general formulas for the trace and divergence of the e.m. tensor. The problem of regularizing the e.m. correlators is strictly connected with (and clarified by) coupling the system to gravity.

\subsection{General formulas for the trace and divergence of the e.m. tensor}

In general let us couple the energy-momentum tensor of a theory to a classical external source $j_{\mu \nu}$. The partition function in terms of $j$ is

$$
\begin{aligned}
Z\left[j^{\mu \nu}\right] & =\left\langle 0\left|\mathcal{T}\left\{e^{\frac{i}{2} \int d x T_{\mu \nu}(x) j^{\mu \nu}(x)}\right\}\right| 0\right\rangle=e^{-i W\left[j_{\mu \nu}\right]} \\
& =\sum_{n=0}^{\infty} \frac{i^{n}}{2^{n} n !} \int \prod_{i=1}^{n} d x_{i} j^{\mu_{i} \nu_{i}}\left(x_{i}\right)\left\langle 0\left|\mathcal{T}\left\{T_{\mu_{1} \nu_{1}}\left(x_{1}\right) \ldots T_{\mu_{n} \nu_{n}}\left(x_{n}\right)\right\}\right| 0\right\rangle,
\end{aligned}
$$


where the symbol $\mathcal{T}$ denotes a time-ordered product. The generating functional of connected Green functions is ${ }^{1}$

$$
W\left[j^{\mu \nu}\right]=\sum_{n=1}^{\infty} \frac{i^{n+1}}{2^{n} n !} \int \prod_{i=1}^{n} d x_{i} j^{\mu_{i} \nu_{i}}\left(x_{i}\right)\left\langle 0\left|\mathcal{T}\left\{T_{\mu_{1} \nu_{1}}\left(x_{1}\right) \cdots T_{\mu_{n} \nu_{n}}\left(x_{n}\right)\right\}\right| 0\right\rangle_{c} .
$$

We will denote the full one-loop e.m. tensor by

$$
\left\langle\left\langle T_{\mu \nu}(x)\right\rangle\right\rangle=\left.2 \frac{\delta W[j]}{\delta j^{\mu \nu}(x)}\right|_{j^{\mu \nu}=h^{\mu \nu}},
$$

where $h_{\mu \nu}$ is the fluctuation, $g_{\mu \nu}=\eta_{\mu \nu}+h_{\mu \nu}+\ldots$ and $g^{\mu \nu}=\eta^{\mu \nu}-h^{\mu \nu}+\ldots$, with respect to the flat metric $\eta_{\mu \nu}{ }^{2}$ The fluctuation $h_{\mu \nu}$ is the field attached to the external legs in the Feynman diagrams approach. We can reconstruct the full one-loop e.m. tensor as a function of $h_{\mu \nu}$ by means of the formula

$$
\begin{aligned}
\left\langle\left\langle T_{\mu \nu}(x)\right\rangle\right\rangle= & \frac{1}{n !} \sum_{n=1}^{\infty} \int d x_{1} \ldots \int d x_{n} h^{\mu_{1} \nu_{1}}\left(x_{1}\right) \ldots h^{\mu_{n} \nu_{n}}\left(x_{n}\right) \\
& \times \frac{\delta}{\delta h^{\mu_{1} \nu_{1}}\left(x_{1}\right)} \ldots \frac{\delta}{\delta h^{\mu_{n} \nu_{n}}\left(x_{n}\right)}\left\langle\left.\left\langle T_{\mu \nu}(x)\right\rangle\right|_{h=0} .\right.
\end{aligned}
$$

For instance, to first and second order in $h$ the trace is given by

$$
\frac{\delta}{\delta h^{\lambda \rho}(y)}\left\langle\left.\left\langle T_{\mu}^{\mu}(x)\right\rangle\right|_{h=0}=2 i\left\langle 0\left|\mathcal{T}\left\{T_{\mu}^{\mu}(x) T_{\lambda \rho}(y)\right\}\right| 0\right\rangle\right.
$$

and

$$
\begin{aligned}
\frac{\delta}{\delta h^{\lambda \rho}(y)} \frac{\delta}{\delta h^{\alpha \beta}(z)}\left\langle\left.\left\langle T_{\mu}^{\mu}(x)\right\rangle\right|_{h=0}=\right. & -2 i\left(\delta^{(4)}(x-y)+\delta^{(4)}(x-z)\right)\left\langle 0\left|\mathcal{T}\left\{T_{\lambda \rho}(y) T_{\alpha \beta}(z)\right\}\right| 0\right\rangle \\
& +2\left\langle 0\left|\mathcal{T}\left\{T_{\mu}^{\mu}(x) T_{\lambda \rho}(y) T_{\alpha \beta}(z)\right\}\right| 0\right\rangle
\end{aligned}
$$

and the divergence by

$$
\left.\frac{\delta}{\delta h^{\lambda \rho}(y)}\left\langle\left\langle\nabla^{\mu} T_{\mu \nu}(x)\right\rangle\right\rangle\right|_{h=0}=-2 i\left\langle 0\left|\mathcal{T}\left\{\partial^{\mu} T_{\mu \nu}(x) T_{\lambda \rho}(y)\right\}\right| 0\right\rangle
$$

\footnotetext{
${ }^{1}$ It should be understood that $\left\langle 0\left|\mathcal{T}\left\{T_{\mu_{1} \nu_{1}}\left(x_{1}\right) \cdots T_{\mu_{n} \nu_{n}}\left(x_{n}\right)\right\}\right| 0\right\rangle_{c}$ corresponds to the sum of all Feynman diagrams of the theory with $n$ external legs. Notice that this is in general different from the $n$-point function of e.m. tensors computed by applying the Wick theorem, see for instance [12, 13, 21]. For example $\left\langle 0\left|\mathcal{T}\left\{T_{\mu \nu}(x) T_{\rho \sigma}(y)\right\}\right| 0\right\rangle_{c}$ corresponds to the sum of two terms calculated with the Wick theorem, $\left\langle T_{\mu \nu}(x) T_{\rho \sigma}(y)\right\rangle$ and $\left\langle\frac{\delta T_{\mu \nu}}{\delta h^{\rho \sigma}}\right\rangle$. In terms of Feynman diagrams the first one corresponds to a bubble diagram while the second corresponds to a tadpole, both with two external legs. These additional terms containing functional derivatives of the e.m. tensors correspond in configuration space to contributions at coincident points. In the computations that are considered in this paper, when regularized, they will not contribute.

${ }^{2}$ The factor $\frac{1}{2^{n}}$ in (1.1) is motivated by the fact that when we expand the action

$$
S[\eta+h]=S[\eta]+\left.\int d^{d} x \frac{\delta S}{\delta g^{\mu \nu}}\right|_{g=\eta} h^{\mu \nu}+\cdots,
$$

the factor $\left.\frac{\delta S}{\delta g^{\mu \nu}}\right|_{g=\eta}=\frac{1}{2} T_{\mu \nu}$. Another consequence of this fact will be that the presence of vertices with one graviton in Feynman diagrams will correspond to insertions of the operator $\frac{1}{2} T_{\mu \nu}$ in correlation functions.
} 
and

$$
\begin{aligned}
& \left.\frac{\delta}{\delta h^{\lambda \rho}(y)} \frac{\delta}{\delta h^{\alpha \beta}(z)}\left\langle\left\langle\nabla^{\mu} T_{\mu \nu}(x)\right\rangle\right\rangle\right|_{h=0}= \\
& i\left\{\frac{\partial}{\partial x^{(\alpha}}\left[\delta(x-z)\left\langle 0\left|\mathcal{T}\left\{T_{\beta) \nu}(x) T_{\lambda \rho}(y)\right\}\right| 0\right\rangle\right]+\frac{\partial}{\partial x^{(\lambda}}\left[\delta(x-y)\left\langle 0\left|\mathcal{T}\left\{T_{\rho) \nu}(x) T_{\alpha \beta}(z)\right\}\right| 0\right\rangle\right]\right. \\
& +2 \frac{\partial}{\partial x_{\tau}} \delta(x-z) \eta_{\alpha \beta}\left\langle 0\left|\mathcal{T}\left\{T_{\tau \nu}(x) T_{\lambda \rho}(y)\right\}\right| 0\right\rangle+2 \frac{\partial}{\partial x_{\tau}} \delta(x-y) \eta_{\lambda \rho}\left\langle 0\left|\mathcal{T}\left\{T_{\tau \nu}(x) T_{\alpha \beta}(z)\right\}\right| 0\right\rangle \\
& \left.+2 \frac{\partial}{\partial x^{\nu}} \delta(x-z)\left\langle 0\left|\mathcal{T}\left\{T_{\lambda \rho}(y) T_{\alpha \beta}(x)\right\}\right| 0\right\rangle+2 \frac{\partial}{\partial x^{\nu}} \delta(x-y)\left\langle 0\left|\mathcal{T}\left\{T_{\lambda \rho}(x) T_{\alpha \beta}(z)\right\}\right| 0\right\rangle\right\} \\
& +2\left\langle 0\left|\mathcal{T}\left\{\partial^{\mu} T_{\mu \nu}(x) T_{\lambda \rho}(y) T_{\alpha \beta}(z)\right\}\right| 0\right\rangle,
\end{aligned}
$$

respectively, where the delta functions are 4-dimensional and the round brackets indicate symmetrization. These formulas are obtained understanding that gravity is minimally coupled and that the background is flat. If there is a nontrivial background metric, say $g_{\mu \nu}^{(0)}$, then we must insert $\sqrt{g^{(0)}}$ in the integral in the exponent of (1.1) and, for instance, (1.6) would be replaced by

$$
\begin{aligned}
& \left.\frac{1}{\sqrt{g^{(0)}(y)}} \frac{\delta}{\delta h^{\lambda \rho}(y)} \frac{1}{\sqrt{g^{(0)}(z)}} \frac{\delta}{\delta h^{\alpha \beta}(z)}\left\langle\left\langle T_{\mu}^{\mu}(x)\right\rangle\right\rangle\right|_{h=0} \\
& =-2 i\left(\frac{\delta^{(4)}(x-y)}{\sqrt{g^{(0)}(y)}}+\frac{\delta^{(4)}(x-z)}{\sqrt{g^{(0)}(z)}}\right)\left\langle 0\left|\mathcal{T}\left\{T_{\lambda \rho}(y) T_{\alpha \beta}(z)\right\}\right| 0\right\rangle+\left\langle 0\left|\mathcal{T}\left\{T_{\mu}^{\mu}(x) T_{\lambda \rho}(y) T_{\alpha \beta}(z)\right\}\right| 0\right\rangle
\end{aligned}
$$

and (1.8) by a much more complicated formula.

\section{2-point function of e.m. tensors in $2 d$ and trace anomaly}

In this section we regularize the 2-point function of energy-momentum tensors in $2 d$ using the techniques of differential regularization and we derive the very well-known $2 d$ trace anomaly. The ambiguities implicit in the regularization procedure allow us to make manifest the interplay between diffeomorphism and trace anomalies.

Let us consider the 2-point function $\left\langle T_{\mu \nu}(x) T_{\rho \sigma}(0)\right\rangle$. This 2-point function in $2 d$ (i.e. the "bare" 2-point function) is very well-known and is given by ${ }^{3}$

$$
\left\langle T_{\mu \nu}(x) T_{\rho \sigma}(0)\right\rangle=\frac{c / 2}{x^{4}}\left(I_{\mu \rho}(x) I_{\nu \sigma}(x)+I_{\nu \rho}(x) I_{\mu \sigma}(x)-\eta_{\mu \nu} \eta_{\rho \sigma}\right)
$$

where

$$
I_{\mu \nu}(x)=\eta_{\mu \nu}-2 \frac{x_{\mu} x_{\nu}}{x^{2}}
$$

and $c$ is the central charge of the theory. For $x \neq 0$ this 2-point function satisfies the Ward identities

$$
\begin{aligned}
\partial^{\mu}\left\langle T_{\mu \nu}(x) T_{\rho \sigma}(0)\right\rangle & =0, \\
\left\langle T_{\mu}^{\mu}(x) T_{\rho \sigma}(0)\right\rangle & =0 .
\end{aligned}
$$

\footnotetext{
${ }^{3}$ One way of deriving this expression is by using the embedding formalism, see [5], for example.
} 
The result (2.1) is obtained using the symmetry properties of the indices, dimensional analysis and eqs. (2.3) and (2.4).

The 2-point function written above are UV singular for $x \rightarrow 0$, hence this divergence has to be dealt with for the correlator to be well-defined everywhere. In this context the most convenient way to regularize this object is with the technique of differential regularization. The recipe of differential regularization is: given a function $f(x)$ that needs to be regularized, find the most general function $F(x)$ such that $\mathcal{D} F(x)=f(x)$, where $\mathcal{D}$ is some differential operator, and such that the Fourier transform of $\mathcal{D} F(x)$ is well-defined (alternatively $\mathcal{D} F(x)$ has integrable singularities).

In our case we have two guiding principles: the Ward identities and dimensional analysis. Differential regularization tells that our 2-point function should be some differential operator applied to a function, i.e.

$$
\left\langle T_{\mu \nu}(x) T_{\rho \sigma}(0)\right\rangle=\mathcal{D}_{\mu \nu \rho \sigma}(f(x)),
$$

while conservation requires that the differential operator $\mathcal{D}_{\mu \nu \rho \sigma}$ be transverse, i.e.

$$
\partial^{\mu} \mathcal{D}_{\mu \nu \rho \sigma}=\cdots=\partial^{\sigma} \mathcal{D}_{\mu \nu \rho \sigma}=0
$$

The most general transverse operator with four derivatives, symmetric in $\mu, \nu$ and in $\rho, \sigma$ that one can write is

$$
\mathcal{D}_{\mu \nu \rho \sigma}=\alpha \mathcal{D}_{\mu \nu \rho \sigma}^{(1)}+\beta \mathcal{D}_{\mu \nu \rho \sigma}^{(2)}
$$

where

$$
\begin{aligned}
\mathcal{D}_{\mu \nu \rho \sigma}^{(1)}= & \partial_{\mu} \partial_{\nu} \partial_{\rho} \partial_{\sigma}-\left(\eta_{\mu \nu} \partial_{\rho} \partial_{\sigma}+\eta_{\rho \sigma} \partial_{\mu} \partial_{\nu}\right) \square+\eta_{\mu \nu} \eta_{\rho \sigma} \square \square, \\
\mathcal{D}_{\mu \nu \rho \sigma}^{(2)}= & \partial_{\mu} \partial_{\nu} \partial_{\rho} \partial_{\sigma}-\frac{1}{2}\left(\eta_{\mu \rho} \partial_{\nu} \partial_{\sigma}+\eta_{\nu \rho} \partial_{\mu} \partial_{\sigma}+\eta_{\mu \sigma} \partial_{\nu} \partial_{\rho}+\eta_{\nu \sigma} \partial_{\mu} \partial_{\rho}\right) \square \\
& +\frac{1}{2}\left(\eta_{\mu \rho} \eta_{\nu \sigma}+\eta_{\nu \rho} \eta_{\mu \sigma}\right) \square \square .
\end{aligned}
$$

One important fact about these differential operators is that they may not be traceless. Indeed, by taking the trace we find

$$
\eta^{\mu \nu} \mathcal{D}_{\mu \nu \rho \sigma}^{(1)}=\eta^{\mu \nu} \mathcal{D}_{\mu \nu \rho \sigma}^{(2)}=-\left(\partial_{\rho} \partial_{\sigma}-\eta_{\rho \sigma} \square\right) \square
$$

Dimensional analysis tells us that the function $f(x)$ in $(2.5)$ can be at most a function of $\log \mu^{2} x^{2}$ since the 1.h.s. of (2.5) scales like $1 / x^{4}$ and this scaling is already saturated by the differential operator with four derivatives. Notice that we have introduced an arbitrary mass scale $\mu$ to make the argument of the log dimensionless. Let us write the most general ansatz for (2.5):

$$
\begin{aligned}
\left\langle T_{\mu \nu}(x) T_{\rho \sigma}(0)\right\rangle= & \mathcal{D}_{\mu \nu \rho \sigma}^{(1)}\left[\alpha_{1} \log \mu^{2} x^{2}+\alpha_{2}\left(\log \mu^{2} x^{2}\right)^{2}+\cdots\right] \\
& +\mathcal{D}_{\mu \nu \rho \sigma}^{(2)}\left[\beta_{1} \log \mu^{2} x^{2}+\beta_{2}\left(\log \mu^{2} x^{2}\right)^{2}+\cdots\right] .
\end{aligned}
$$


Now our task is to fix the coefficients $\alpha_{i}$ and $\beta_{j}$ for (2.11) to match (2.1) for $x \neq 0$. As it turns out we only need terms up to $\log ^{2}$ (otherwise one cannot avoid logarithmic terms for $x \neq 0$ ) The matching gives us

thus

$$
\alpha_{1}=-\frac{c}{24}-\beta_{1}, \quad \alpha_{2}=-\beta_{2}=-\frac{c}{96},
$$

$$
\left\langle T_{\mu \nu}(x) T_{\rho \sigma}(0)\right\rangle=-\frac{c}{24} \mathcal{D}_{\mu \nu \rho \sigma}^{(1)}\left(\log \mu^{2} x^{2}\right)-\frac{c}{96}\left(\mathcal{D}_{\mu \nu \rho \sigma}^{(1)}-\mathcal{D}_{\mu \nu \rho \sigma}^{(2)}\right)\left(\log \mu^{2} x^{2}\right)^{2} .
$$

Notice that $\beta_{1}$ is absent in the final result. Indeed, the term with coefficient $\beta_{1}$ is

$$
-\left(\mathcal{D}_{\mu \nu \rho \sigma}^{(1)}-\mathcal{D}_{\mu \nu \rho \sigma}^{(2)}\right)\left(\log \mu^{2} x^{2}\right)
$$

and this term identically vanishes in $2 d$. If we take the trace of $(2.12)$ we find that

$$
\left\langle T_{\mu}^{\mu}(x) T_{\rho \sigma}(0)\right\rangle=-\frac{c}{48} \eta^{\mu \nu} \mathcal{D}_{\mu \nu \rho \sigma}^{(1)}\left(\log \mu^{2} x^{2}\right)=\frac{c}{48}\left(\partial_{\rho} \partial_{\sigma}-\eta_{\rho \sigma} \square\right) \square \log \mu^{2} x^{2} .
$$

These terms have support only at $x=0$, for in $2 d$ the d'Alembertian of a log is a delta function, more precisely

$$
\square \log \mu^{2} x^{2}=4 \pi \delta^{2}(x) .
$$

Therefore we find the anomalous Ward identity

$$
\left\langle T_{\mu}^{\mu}(x) T_{\rho \sigma}(y)\right\rangle=c \frac{\pi}{12}\left(\partial_{\rho} \partial_{\sigma}-\eta_{\rho \sigma} \square\right) \delta^{2}(x-y),
$$

If we consider our theory in the presence of a background metric $g$ which is a perturbation of flat spacetime, i.e. $g_{\rho \sigma}(y)=\eta_{\rho \sigma}+h_{\rho \sigma}(y)+\cdots$, eq. (2.15) gives rise to the lowest contribution to the 'full one-loop' trace of the e.m. tensor, namely

$$
\left\langle\left\langle T_{\mu}^{\mu}\right\rangle\right\rangle=c \frac{\pi}{12}\left(\partial_{\mu} \partial_{\nu}-\eta_{\mu \nu} \square\right) h^{\mu \nu}
$$

which coincides with the lowest contribution of the expansion in $h$ of the Ricci scalar, i.e.

$$
R=\left(\partial_{\mu} \partial_{\nu}-\eta_{\mu \nu} \square\right) h^{\mu \nu}+\mathcal{O}\left(h^{2}\right) .
$$

Covariance requires that the higher order corrections in $h$ to the 'full one-loop' trace of the e.m. tensor in the presence of a background metric $g$ to be such that we recover the covariant expression

$$
\left\langle\left\langle T_{\mu}^{\mu}\right\rangle\right\rangle=c \frac{\pi}{12} R
$$

For a free chiral fermion $c=1 / 4 \pi^{2}$, vide section 4 or appendix A. We are authorized to use the covariant expression (2.18) because the energy-momentum tensor is conserved (there are no diffemorphism anomalies).

Using the above results it is easy to verify the Callan-Symanzik equation for the 2point function (2.12). The Callan-Symanzik differential operator reduces to the logarithmic derivative with respect to $\mu$, because both beta functions and anomalous dimensions vanish in the case we are considering. We get

$$
\mu \frac{\partial}{\partial \mu}\left\langle T_{\mu \nu}(x) T_{\rho \sigma}(0)\right\rangle \sim\left(\mathcal{D}_{\mu \nu \rho \sigma}^{(1)}-\mathcal{D}_{\mu \nu \rho \sigma}^{(2)}\right)\left(\log \mu^{2} x^{2}\right)=0 .
$$

We see that requiring that the regularized correlator satisfies conservation at $x=0$ implies the appearance of a trace anomaly. However this is not the end of the story, since there are ambiguities in the regularization process we have so far disregarded. 


\subsection{Ambiguities}

The ambiguity arises from the fact that we can add to (2.12) terms that have support only in $x=0$. The most general modification of the parity-even part that would affect only its expression for $x=0$ is given by

$$
\begin{aligned}
A_{\mu \nu \rho \sigma}= & A\left(\eta_{\mu \nu} \partial_{\rho} \partial_{\sigma}+\eta_{\rho \sigma} \partial_{\mu} \partial_{\nu}\right) \square \log \mu^{2} x^{2} \\
& +B\left(\eta_{\mu \rho} \partial_{\nu} \partial_{\sigma}+\eta_{\nu \rho} \partial_{\mu} \partial_{\sigma}+\eta_{\mu \sigma} \partial_{\nu} \partial_{\rho}+\eta_{\nu \sigma} \partial_{\mu} \partial_{\rho}\right) \square \log \mu^{2} x^{2} \\
& +C\left(\eta_{\mu \rho} \eta_{\nu \sigma}+\eta_{\nu \rho} \eta_{\mu \sigma}\right) \square \square \log \mu^{2} x^{2} \\
& +D \eta_{\mu \nu} \eta_{\rho \sigma} \square \square \log \mu^{2} x^{2} .
\end{aligned}
$$

We remark that this term is in general neither conserved nor traceless

$$
\begin{aligned}
\partial^{\mu} A_{\mu \nu \rho \sigma}= & 4 \pi\left((A+2 B) \partial_{\nu} \partial_{\rho} \partial_{\sigma}+(A+D) \eta_{\rho \sigma} \partial_{\nu} \square\right. \\
& \left.+(B+C)\left(\eta_{\rho \nu} \partial_{\sigma} \square+\eta_{\sigma \nu} \partial_{\rho} \square\right)\right) \delta^{(2)}(x) \\
A_{\mu \rho \sigma}^{\mu}= & 4 \pi\left((2 A+4 B) \partial_{\rho} \partial_{\sigma}+(A+2 C+2 D) \eta_{\rho \sigma} \square\right) \delta^{(2)}(x)
\end{aligned}
$$

We notice that by imposing (2.21) to vanish imply that also (2.22) will vanish. We may wonder whether using this ambiguity we can cancel the trace anomaly. This can certainly be done by choosing $2 A+4 B=-A-2 C-2 D$ and adjusting the overall coefficient. But this operation gives rise to a diffeomorphism anomaly. Its form is far from appealing and not particularly illuminating, so we do not write it down (see however $[23,24]$ ). In other words the anomaly (2.18) is a non-trivial cocycle of the overall symmetry diffeomorphisms plus Weyl transformations. As was discussed in $[23,24]$ it may take different forms, either as a pure diffeomorphism anomaly or a pure trace anomaly. In general both components may be nonvanishing. It is obvious that, in practice, it is more useful to preserve diffeomorphism invariance, so that the cocycle takes the form (2.18).

\section{Parity-odd terms in $2 d$}

In this section we compute all possible "bare" parity-odd terms in the 2-point function of the energy-momentum tensor in $2 d$. We follow three methods, the first two are general while the third is based on a specific model. Needless to say all methods give the same results up to ambiguities.

\subsection{Using symmetries}

The first method is very simple-minded, it consists in writing the most general expression $\mathcal{T}_{\mu \nu \rho \sigma}^{\text {odd }}(x)$ linear in the antisymmetric tensor $\epsilon_{\alpha \beta}$ with the right dimensions which is symmetric and traceless in $\mu, \nu$ and $\rho, \sigma$ separately, is symmetric in the exchange $(\mu, \nu) \leftrightarrow(\rho, \sigma)$, and is conserved. The calculation is tedious but straightforward. The result is as follows. Let us define

$$
T_{\mu \nu \rho \sigma}=\frac{1}{x^{4}}\left(I_{\mu \rho}(x) I_{\nu \sigma}(x)+I_{\mu \sigma}(x) I_{\nu \rho}(x)-\eta_{\mu \nu} \eta_{\rho \sigma}\right),
$$


and

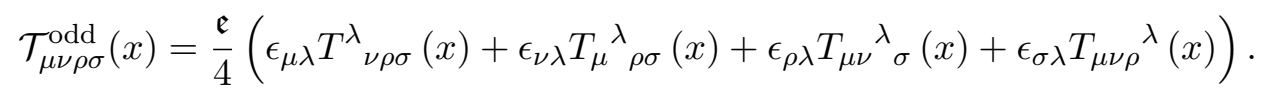

where $\mathfrak{e}$ is an undetermined constant. We assume (3.2) to represent $\left\langle T_{\mu \nu}(x) T_{\rho \sigma}(0)\right\rangle_{\text {odd }}$. It satisfies all the desired properties (it is traceless and conserved). In order to make sure that it is conformal covariant, we have to check that it is chirally split. To this end we introduce the light-cone coordinates $x_{ \pm}=x^{0} \pm x^{1}$. It is not hard to verify that

$$
\left\langle T_{++}(x) T_{--}(0)\right\rangle_{\text {odd }}=0 .
$$

\subsection{The embedding formalism}

The second method is the embedding formalism [5,6], which consists in using the fact that conformal covariance in $d$ dimensions can be linearly realized in $d+2$. After constructing a covariant expression in $d+2$ one projects to $d$ dimensional Minkowski space. In particular for $d=2$ the method works as follows. We write the most general parity-odd contribution to the 2-point function of a symmetric 2-tensor in $4 d$ which, in addition, is transverse:

$$
\left\langle T_{A B}(X) T_{C D}(Y)\right\rangle_{\text {odd }}=\frac{1}{(X \cdot Y)^{2}}\left[\epsilon_{A I C J} \frac{X^{I} Y^{J}}{X \cdot Y}\left(\eta_{B D}-\frac{X_{D} Y_{B}}{X \cdot Y}\right)+A \leftrightarrow B\right]+C \leftrightarrow D .
$$

This term is symmetric on $A, B$ and $C, D$ and is transverse with respect to $X_{A}, X_{B}$, $Y_{C}$ and $Y_{D}$. Our next step is to project this quantity to $2 d$. The projected correlator is given by

$$
\left\langle T_{\mu \nu}(x) T_{\rho \sigma}(y)\right\rangle_{\text {odd }}=\frac{\partial X^{A}}{\partial x^{\mu}} \frac{\partial X^{B}}{\partial x^{\nu}} \frac{\partial Y^{C}}{\partial y^{\rho}} \frac{\partial Y^{D}}{\partial y^{\sigma}}\left\langle T_{A B}(X) T_{C D}(Y)\right\rangle_{\text {odd }} .
$$

We recall that

$$
\frac{\partial X^{A}}{\partial x^{\mu}}=\delta_{-}^{A} 2 x_{\mu}+\delta_{\mu}^{A} \equiv\left(0,2 x_{\mu}, \delta_{\mu}^{a}\right), \quad A=+,-, a .
$$

The contractions with the $\epsilon$-tensor give rise to a determinant, namely

$$
\epsilon_{A I C J} \frac{\partial X^{A}}{\partial x^{\mu}} X^{I} \frac{\partial Y^{C}}{\partial y^{\rho}} Y^{J} \equiv\left|\begin{array}{cccc}
0 & 1 & 0 & 1 \\
2 x_{\mu} & x^{2} & 2 y_{\rho} & y^{2} \\
\delta_{\mu}^{a} & x^{i} & \delta_{\rho}^{c} & y^{j}
\end{array}\right|
$$

The translational invariance of the problem allows us to rewrite it in the form

$$
\left|\begin{array}{cccc}
0 & 1 & 0 & 1 \\
2(x-y)_{\mu} & (x-y)^{2} & 0 & 0 \\
\delta_{\mu}^{a} & (x-y)^{i} & \delta_{\rho}^{c} & 0
\end{array}\right|=-\left|\begin{array}{ccc}
2(x-y)_{\mu} & (x-y)^{2} & 0 \\
\delta_{\mu}^{a} & (x-y)^{i} & \delta_{\rho}^{c}
\end{array}\right| .
$$

For convenience, let us relabel $x-y \rightarrow x$. This determinant is straightforward to compute and it gives us

$$
-\left|\begin{array}{ccc}
2 x_{\mu} & x^{2} & 0 \\
\delta_{\mu}^{a} & x^{i} & \delta_{\rho}^{c}
\end{array}\right|=-\left(2 x_{\mu}\left|x^{i} \delta_{\rho}^{c}\right|-x^{2}\left|\delta_{\mu}^{a} \delta_{\rho}^{c}\right|\right)=-\left(2 x_{\mu} \epsilon_{\alpha \rho} x^{\alpha}-x^{2} \epsilon_{\mu \rho}\right) .
$$


Thus, the projected correlator is given by

$$
\left\langle T_{\mu \nu}(x) T_{\rho \sigma}(0)\right\rangle_{\text {odd }}=\frac{e}{x^{4}}\left[\epsilon_{\alpha \rho}\left(\delta_{\mu}^{\alpha}-2 \frac{x_{\mu} x^{\alpha}}{x^{2}}\right)\left(\eta_{\nu \sigma}-2 \frac{x_{\nu} x_{\sigma}}{x^{2}}\right)+\mu \leftrightarrow \nu\right]+\rho \leftrightarrow \sigma .
$$

In terms of $I_{\mu \nu}(x)$ we have

$$
\begin{aligned}
\left\langle T_{\mu \nu}(x) T_{\rho \sigma}(0)\right\rangle_{\mathrm{odd}}= & \frac{e}{x^{4}}\left[\epsilon_{\alpha \rho}\left(I_{\mu}^{\alpha}(x) I_{\nu \sigma}(x)+I_{\nu}^{\alpha}(x) I_{\mu \sigma}(x)\right)\right. \\
& \left.+\epsilon_{\alpha \sigma}\left(I_{\mu}^{\alpha}(x) I_{\nu \rho}(x)+I_{\nu}^{\alpha}(x) I_{\mu \rho}(x)\right)\right]
\end{aligned}
$$

This correlator satisfies both tracelessness and conservation, as it can be verified by a direct computation, but it is not symmetric under the exchange of $\mu, \nu$ with $\rho, \sigma$. Thus, our final expression is (3.11) symmetrized in $(\mu, \nu) \leftrightarrow(\rho, \sigma)$ :

$$
\begin{aligned}
\left\langle T_{\mu \nu}(x) T_{\rho \sigma}(0)\right\rangle_{\text {odd }}= & \frac{e}{x^{4}}\left[\epsilon_{\alpha \mu}\left(I_{\rho}^{\alpha}(x) I_{\nu \sigma}(x)+I_{\sigma}^{\alpha}(x) I_{\nu \rho}(x)\right)\right. \\
& +\epsilon_{\alpha \nu}\left(I_{\rho}^{\alpha}(x) I_{\mu \sigma}(x)+I_{\sigma}^{\alpha}(x) I_{\mu \rho}(x)\right) \\
& +\epsilon_{\alpha \rho}\left(I_{\mu}^{\alpha}(x) I_{\nu \sigma}(x)+I_{\nu}^{\alpha}(x) I_{\mu \sigma}(x)\right) \\
& \left.+\epsilon_{\alpha \sigma}\left(I_{\mu}^{\alpha}(x) I_{\nu \rho}(x)+I_{\nu}^{\alpha}(x) I_{\mu \rho}(x)\right)\right] .
\end{aligned}
$$

From (3.12) we notice a tensorial structure very similar to the parity-even part of the 2-point function of $T_{\mu \nu}$, namely

$$
T_{\mu \nu \rho \sigma}(x)=\frac{1}{x^{4}}\left(I_{\mu \rho}(x) I_{\nu \sigma}(x)+I_{\nu \rho}(x) I_{\mu \sigma}(x)-\eta_{\mu \nu} \eta_{\rho \sigma}\right)
$$

and it turns out that we may write (3.12) in terms of the partity-even part, i.e.

$$
\left\langle T_{\mu \nu}(x) T_{\rho \sigma}(0)\right\rangle_{\text {odd }}=\frac{e}{2}\left(\epsilon_{\alpha \mu} T_{\nu \rho \sigma}^{\alpha}(x)+\epsilon_{\alpha \nu} T_{\mu \rho \sigma}^{\alpha}(x)+\epsilon_{\alpha \rho} T_{\mu \nu}^{\alpha}{ }_{\sigma}^{\alpha}(x)+\epsilon_{\alpha \rho} T_{\mu \nu \rho}^{\alpha}(x)\right) .
$$

This result looks different from (3.2) but it is not hard to show that, for $x \neq 0$, they are proportional: $\mathfrak{e}=\frac{3}{4} e$

Still another method to derive the same result is to use a free fermion model. This is deferred to appendix A.

\subsection{Differential regularization of the parity-odd part}

The task of regularizing the parity-odd terms is very much simplified by the fact that we are able to write them in terms of the parity-even part, see (3.14). We can therefore use the same regularization as in section 2. Let us start by the regularization that preserves diffeomorphisms for the parity-even part, eq. (2.12):

$$
T_{\mu \nu \rho \sigma}(x)=-\frac{1}{12} \mathcal{D}_{\mu \nu \rho \sigma}^{(1)}\left(\log \mu^{2} x^{2}\right)-\frac{1}{48}\left(\mathcal{D}_{\mu \nu \rho \sigma}^{(1)}-\mathcal{D}_{\mu \nu \rho \sigma}^{(2)}\right)\left(\log \mu^{2} x^{2}\right)^{2} .
$$

Regularizing (3.14) with (3.15) leads to a trace anomaly

$$
\left\langle T_{\mu}^{\mu}(x) T_{\rho \sigma}(0)\right\rangle_{\text {odd }}=\frac{\pi e}{24}\left(\epsilon_{\rho \alpha} \partial^{\alpha} \partial_{\sigma}+\epsilon_{\sigma \alpha} \partial^{\alpha} \partial_{\rho}\right) \delta^{2}(x),
$$


and a diffeomorphism anomaly

$$
\partial^{\mu}\left\langle T_{\mu \nu}(x) T_{\rho \sigma}(0)\right\rangle_{\text {odd }}=\frac{\pi e}{24} \epsilon_{\nu \alpha} \partial^{\alpha}\left(\eta_{\rho \sigma} \square-\partial_{\rho} \partial_{\sigma}\right) \delta^{2}(x) .
$$

In the presence of a background metric $g$ the anomalous Ward-Identities (3.16) and (3.17) give rise to the following 'full one-loop' functions

$$
\begin{aligned}
\left\langle\left\langle T_{\mu}^{\mu}(x)\right\rangle\right\rangle & =\frac{\pi e}{24} \epsilon^{\lambda \alpha} \partial_{\alpha}\left(g^{\rho \sigma} \partial_{\lambda} g_{\rho \sigma}+g^{\rho \sigma} \partial_{\rho} g_{\lambda \sigma}\right), \\
\left\langle\left\langle\nabla^{\mu} T_{\mu \nu}(x)\right\rangle\right\rangle & =\frac{\pi e}{24} \epsilon_{\nu \alpha} \partial^{\alpha} R .
\end{aligned}
$$

The second is the well-known covariant form of the diffeomorphism anomaly. The consistent form of the same anomaly is

$$
\left\langle\left\langle\nabla^{\mu} T_{\mu \nu}(x)\right\rangle\right\rangle \sim \epsilon^{\mu \rho} \partial_{\mu} \partial_{\alpha} \Gamma_{\rho \nu}^{\alpha} .
$$

We remark however that in $2 d$ the two forms (3.19) and (3.20) collapse to the same form to the lowest order, since

$$
2 \epsilon_{\mu \nu} \partial^{\mu}\left(\partial_{\alpha} \partial_{\beta}-\eta_{\alpha \beta} \square\right)=\epsilon_{\mu \alpha}\left(\partial^{\mu} \partial_{\nu} \partial_{\beta}-\eta_{\nu \beta} \partial^{\mu} \square+(\alpha \leftrightarrow \beta)\right)
$$

We see that, in any case, the diffeomorphism anomaly is accompanied by the a trace anomaly.

\subsection{Ambiguities in the parity-odd part}

We know that the regularization used above is not the ultimate one, because there are ambiguities. They entail a modification of the parity-odd part given by

$$
A_{\mu \nu \rho \sigma}^{\text {odd }}=\epsilon_{\alpha \mu} A_{\nu \rho \sigma}^{\alpha}+\epsilon_{\alpha \nu} A_{\mu \rho \sigma}^{\alpha}+\epsilon_{\alpha \rho} A_{\mu \nu \sigma_{\sigma}}^{\alpha}+\epsilon_{\alpha \rho} A_{\mu \nu \rho}{ }^{\alpha},
$$

where the r.h.s. is written in terms of (2.20), which explicitly is

$$
\begin{aligned}
A_{\mu \nu \rho \sigma}^{\text {odd }}= & A\left[\eta_{\mu \nu}\left(\epsilon_{\rho \alpha} \partial^{\alpha} \partial_{\sigma}+\epsilon_{\sigma \alpha} \partial^{\alpha} \partial_{\rho}\right)+\eta_{\rho \sigma}\left(\epsilon_{\mu \alpha} \partial^{\alpha} \partial_{\nu}+\epsilon_{\nu \alpha} \partial^{\alpha} \partial_{\mu}\right)\right] \square \log \mu^{2} x^{2} \\
& +B\left[\epsilon_{\mu \alpha}\left(\eta_{\nu \rho} \partial^{\alpha} \partial_{\sigma}+\eta_{\nu \sigma} \partial^{\alpha} \partial_{\rho}\right)+\epsilon_{\nu \alpha}\left(\eta_{\mu \rho} \partial^{\alpha} \partial_{\sigma}+\eta_{\mu \sigma} \partial^{\alpha} \partial_{\rho}\right)\right. \\
& \left.+\epsilon_{\rho \alpha}\left(\eta_{\sigma \mu} \partial^{\alpha} \partial_{\nu}+\eta_{\sigma \nu} \partial^{\alpha} \partial_{\mu}\right)+\epsilon_{\sigma \alpha}\left(\eta_{\rho \mu} \partial^{\alpha} \partial_{\nu}+\eta_{\rho \nu} \partial^{\alpha} \partial_{\mu}\right)\right] \square \log \mu^{2} x^{2} .
\end{aligned}
$$

The trace and the divergence of (3.22) are given by:

$$
\begin{aligned}
\eta^{\mu \nu} A_{\mu \nu \rho \sigma}= & 8 \pi(A+2 B)\left(\epsilon_{\rho \alpha} \partial^{\alpha} \partial_{\sigma}+\epsilon_{\sigma \alpha} \partial^{\alpha} \partial_{\rho}\right) \delta^{2}(x), \\
\partial^{\mu} A_{\mu \nu \rho \sigma}= & \pi\left(B \eta_{\nu \rho} \square+(A+B) \partial_{\nu} \partial_{\rho}\right) \epsilon_{\sigma \alpha} \partial^{\alpha} \delta^{2}(x) \\
& +4 \pi\left(B \eta_{\nu \sigma} \square+(A+B) \partial_{\nu} \partial_{\sigma}\right) \epsilon_{\rho \alpha} \partial^{\alpha} \delta^{2}(x) \\
& +4 \pi\left(A \eta_{\rho \sigma} \square+2 B \partial_{\rho} \partial_{\sigma}\right) \epsilon_{\nu \alpha} \partial^{\alpha} \delta^{2}(x) .
\end{aligned}
$$

Using these ambiguities we can recast the expressions (3.16) and (3.17) in the form

$$
\begin{aligned}
\left\langle T_{\mu}^{\mu}(x) T_{\rho \sigma}(0)\right\rangle_{\text {odd }}= & \left(8 \pi(A+2 B)+\frac{\pi e}{24}\right)\left(\epsilon_{\rho \alpha} \partial^{\alpha} \partial_{\sigma}+\epsilon_{\sigma \alpha} \partial^{\alpha} \partial_{\rho}\right) \delta^{2}(x), \\
\partial^{\mu}\left\langle T_{\mu \nu}(x) T_{\rho \sigma}(0)\right\rangle_{\text {odd }}= & 4 \pi\left(B \eta_{\nu \rho} \square+(A+B) \partial_{\nu} \partial_{\rho}\right) \epsilon_{\sigma \alpha} \partial^{\alpha} \delta^{2}(x) \\
& +4 \pi\left(B \eta_{\nu \sigma} \square+(A+B) \partial_{\nu} \partial_{\sigma}\right) \epsilon_{\rho \alpha} \partial^{\alpha} \delta^{2}(x) \\
& +\epsilon_{\nu \alpha} \partial^{\alpha}\left(\left(4 \pi A+\frac{\pi e}{24}\right) \eta_{\rho \sigma} \square+\left(8 \pi B-\frac{\pi e}{24}\right) \partial_{\rho} \partial_{\sigma}\right) \delta^{2}(x) .
\end{aligned}
$$


If we impose that (3.25) is zero we find

$$
A=-\frac{e}{192}-2 B
$$

which implies that (3.26) takes the form

$$
\begin{aligned}
\partial^{\mu}\left\langle T_{\mu \nu}(x) T_{\rho \sigma}(0)\right\rangle_{\text {odd }}= & 4 \pi\left[B \eta_{\nu \rho} \square-\left(\frac{e}{192}+B\right) \partial_{\nu} \partial_{\rho}\right] \epsilon_{\sigma \alpha} \partial^{\alpha} \delta^{2}(x) \\
& +4 \pi\left[B \eta_{\nu \sigma} \square-\left(\frac{e}{192}+B\right) \partial_{\nu} \partial_{\sigma}\right] \epsilon_{\rho \alpha} \partial^{\alpha} \delta^{2}(x) \\
& -\epsilon_{\nu \alpha} \partial^{\alpha}\left[\left(\frac{\pi e}{48}+8 \pi B\right) \eta_{\rho \sigma} \square-\left(8 \pi B-\frac{\pi e}{24}\right) \partial_{\rho} \partial_{\sigma}\right] \delta^{2}(x)
\end{aligned}
$$

The choice (3.27) allows us to eliminate the trace anomaly (3.18) but by doing so the diffeo anomaly becomes (3.28), which will not imply a covariant expression for $\left\langle\left\langle T_{\mu \nu}\right\rangle\right\rangle$ for any choice of $B$. Thus, the most general regularization that one can write is given by the equations (3.25) and (3.26). An important point of (3.26) is that there is no choice of $A$ and $B$ for which it is zero, hence inevitably we will have a diffeomorphism anomaly, unless the overall factor $e=0$, which depends of course on the specific model.

\section{The Feynman diagrams method in $2 d$}

It is interesting and instructive to derive the results above using Feynman diagrams. There is only one non-trivial contribution that comes from the bubble diagram with one incoming and one outgoing line with momentum $k$ and an internal momentum $p$ (see figure 1). The pertinent Feynman rule is

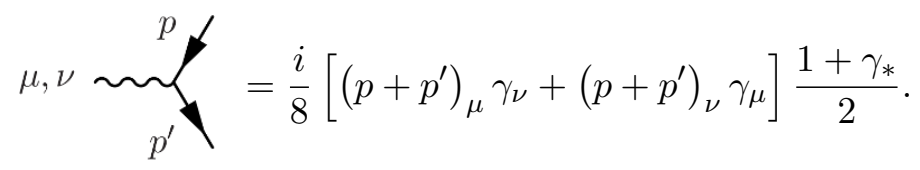

The relevant 2-point function is ${ }^{4}$

$$
\left\langle T_{\mu \nu}(x) T_{\lambda \rho}(y)\right\rangle=4 \int \frac{d^{2} k}{(2 \pi)^{2}} e^{-i k(x-y)} \mathcal{T}_{\mu \nu \lambda \rho}(k)
$$

with

$$
\mathcal{T}_{\mu \nu \lambda \rho}(k)=-\frac{1}{64} \int \frac{d^{2} k}{(2 \pi)^{2}} \operatorname{tr}\left(\frac{1}{\not p}(2 p-k)_{\mu} \gamma_{\nu} \frac{1}{\not p-\not k}(2 p-k)_{\lambda} \gamma_{\rho} \frac{1+\gamma_{*}}{2}\right)+\left\{\begin{array}{l}
\mu \leftrightarrow \nu \\
\lambda \leftrightarrow \rho
\end{array}\right\} .
$$

Taking the trace and regularizing by introducing extra components of the momentum running around the loop, $p \rightarrow p+\ell\left(\ell=\ell_{2}, \ldots, \ell_{\delta+2}\right)$, we get

$$
\begin{aligned}
\mathcal{T}_{\mu \lambda \rho}^{\mu}(k)= & -\frac{1}{32} \int \frac{d^{2} p}{(2 \pi)^{2}} \int \frac{d^{\delta} \ell}{(2 \pi)^{\delta}} \operatorname{tr}\left(\frac{\not p+\ell}{p^{2}-\ell^{2}}(2 \not p+2 \ell-\not q)\right. \\
& \left.\frac{\not p+\ell-\not k}{(p-k)^{2}-\ell^{2}}(2 p-k)_{\lambda} \gamma_{\rho} \frac{1+\gamma_{*}}{2}\right)
\end{aligned}
$$




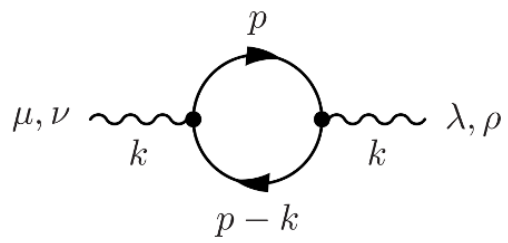

Figure 1. The relevant Feynman diagram for the computation.

and the symmetrization $\lambda \leftrightarrow \rho$ is understood from now on. Introducing, as usual, a Feynman parametrization of the integral in (4.4) and using the results in appendix (C) one finally gets for the even part

$$
\left(\mathcal{T}_{\text {even }}\right)_{\mu \lambda \rho}^{\mu}(k)=\frac{1}{192 \pi}\left(\eta_{\lambda \rho} k^{2}+k_{\lambda} k_{\rho}\right),
$$

which corresponds to the trace anomaly

$$
\left\langle\left\langle T_{\mu}^{\mu}\right\rangle\right\rangle=-\frac{1}{48 \pi}\left(\square h+\partial_{\lambda} \partial_{\rho} h^{\lambda \rho}\right)+\mathcal{O}\left(h^{2}\right) .
$$

For the odd part we get instead

$$
\left(\mathcal{T}_{\text {odd }}\right)_{\mu \lambda \rho}^{\mu}(k)=-\frac{1}{192 \pi}\left(\epsilon_{\rho}^{\sigma} k_{\sigma} k_{\lambda}+\left(\epsilon_{\lambda}^{\sigma} k_{\sigma} k_{\rho}\right),\right.
$$

which corresponds to the trace anomaly

$$
\left\langle\left\langle T_{\mu}^{\mu}\right\rangle\right\rangle=\frac{1}{24 \pi} \epsilon_{\rho}^{\sigma} \partial_{\sigma} \partial_{\lambda} h^{\lambda \rho}+\mathcal{O}\left(h^{2}\right) .
$$

The trace anomaly (4.6) is not the expected covariant one. The only possible explanation is that our regularization has broken diffeomorphism invariance. In order to check that we have to compute the divergence of the energy-momentum tensor with the same method. The relevant Feynman diagram contribution is (after regularization)

$$
\begin{aligned}
\mathcal{D}_{\nu \lambda \rho}(k)= & -\frac{1}{64} \int \frac{d^{2} p}{(2 \pi)^{2}} \int \frac{d^{\delta} \ell}{(2 \pi)^{\delta}} \\
& \operatorname{tr}\left(\frac{\not p+\ell}{p^{2}-\ell^{2}}(2 p-k)_{\mu} k^{\mu} \gamma_{\nu} \frac{\not p+\ell-\not k}{(p-k)^{2}-\ell^{2}}(2 p-k)_{\lambda} \gamma_{\rho} \frac{1+\gamma_{*}}{2}\right. \\
& \left.+\frac{\not p+\ell}{p^{2}-\ell^{2}}(2 p-k)_{\nu} \not k \frac{\not p+\ell-\not k}{(p-k)^{2}-\ell^{2}}(2 p-k)_{\lambda} \gamma_{\rho} \frac{1+\gamma_{*}}{2}\right) .
\end{aligned}
$$

Explicit evaluation gives for the even part

$$
\left(\mathcal{D}_{\text {even }}\right)_{\nu \lambda \rho}(k)=-\frac{1}{96 \pi} \eta_{\lambda \rho} k_{\nu} k^{2},
$$

\footnotetext{
${ }^{4}$ The factor of 4 in (4.2) is produced by the fact that the vertex (4.1) corresponds to the insertion of $\frac{1}{2} T_{\mu \nu}$, not simply $T_{\mu \nu}$, in the correlator, as explained in the footnote in (1.1).
} 
which corresponds to the diffeomorphism anomaly

$$
\nabla^{\mu}\left\langle\left\langle T_{\mu \nu}\right\rangle\right\rangle=\frac{1}{12 \pi} \xi^{\nu} \partial_{\nu} \square h+\mathcal{O}\left(h^{2}\right) .
$$

For the odd part we get instead

$$
\left(\mathcal{D}_{\text {odd }}\right)_{\nu \lambda \rho}(k)=-\frac{1}{192 \pi} k^{\sigma}\left(\epsilon_{\sigma \rho} \eta_{\nu \lambda}-\epsilon_{\nu \rho} k_{\lambda}\right) k^{2}+\{\lambda \leftrightarrow \rho\},
$$

which corresponds to the anomaly

$$
\nabla^{\mu}\left\langle\left\langle T_{\mu \nu}\right\rangle\right\rangle=-\frac{1}{96 \pi} \epsilon^{\sigma \rho}\left(\partial_{\sigma} \partial_{\lambda} \partial_{\nu} h_{\rho}^{\lambda}-\partial_{\sigma} \square h_{\rho \nu}\right) .
$$

Using the lowest order Weyl transformation

$$
\delta_{\omega} h_{\mu \nu}=2 \omega \eta_{\mu \nu}
$$

and diffeo transformation

$$
\delta_{\xi} h_{\mu \nu}=\partial_{\mu} \xi_{\nu}+\partial_{\nu} \xi_{\mu},
$$

it is easy to prove that the consistency relations

$$
\delta_{\omega} \mathcal{A}_{\omega}=0, \quad \delta_{\xi} \mathcal{A}_{\omega}+\delta_{\xi} \mathcal{A}_{\omega}=0, \quad \delta_{\xi} \mathcal{A}_{\xi}=0,
$$

hold, where

$$
\mathcal{A}_{\omega}=-\int d^{2} x \omega\left\langle\left\langle T_{\mu}^{\mu}\right\rangle\right\rangle, \quad \text { and } \quad \mathcal{A}_{\xi}=\int d^{2} x \xi^{\nu} \nabla^{\mu}\left\langle\left\langle T_{\mu \nu}\right\rangle\right\rangle .
$$

For the even part $\mathcal{A}^{(e)}$ it is possible to add a counterterm to the action and restore covariance. The couterterm is

$$
\mathcal{C}=-\frac{1}{96 \pi} \int d^{2} x h \square h
$$

After this operation the divergence of the e.m. tensor vanishes and the trace anomaly becomes

$$
\mathcal{A}_{\omega}^{(e)} \rightarrow \mathcal{A}_{\omega}^{(e)}+\delta_{\omega} \mathcal{C}=\frac{1}{48 \pi} \int d^{2} x \omega\left(\partial_{\lambda} \partial_{\rho} h^{\lambda \rho}-\square h\right),
$$

which is the expected one (see above).

Similarly the parity-odd anomalies (4.7) and (4.13) satisfy the consistency relations (4.16). One can add an odd counterterm to eliminate the odd trace anomaly but this is definitely a less interesting operation.

The results obtained in this section are well-known. The methods we have used to derive them teach us important lessons. The first concerns dimensional regularization. If not explicitly stated it is often understood in the literature that dimensional regularization of Feynman diagrams leads to covariant results. We have seen explicitly that this is not true, and a reconstruction of covariance with counterterms is inevitable. In view of the 
discussion on 3-points correlator of the e.m. tensor in section 6.4 we notice that the piece of $(4.4)$

$$
\Delta \mathcal{T}_{\mu \lambda \rho}^{\mu}(k)=-\frac{1}{8} \int \frac{d^{2} p}{(2 \pi)^{2}} \int \frac{d^{\delta} \ell}{(2 \pi)^{\delta}} \operatorname{tr}\left(\frac{\not p+\ell}{p^{2}-\ell^{2}} 2 \ell \frac{\not p+\ell-\not k}{(p-k)^{2}-\ell^{2}}(2 p-k)_{\lambda} \gamma_{\rho} \frac{1+\gamma_{*}}{2}\right)
$$

contributes in an essential way to both even and odd anomalies. Without this piece the result of the calculation would be inconsistent. It marks the difference between first regularizing and then taking the trace of the e.m. tensor or first taking the trace and then regularizing. From the above it is obvious that the second procedure is the correct one. In other words every irreducible Lorentz component of tensors must be regularized separately. This is the second important lesson. We will return to this point also in the final section.

\section{2-point correlator of e.m. tensors in $4 d$}

In this section we are going to discuss the 2-point correlator of the e.m. tensors in $4 d$. The expression in coordinate representation is well-known. We would like here to regularize it with the differential regularization method, and, later on, compare it with the expression obtained in momentum space with Feynman diagram techniques.

\subsection{Differential regularization of the correlator}

The unregulated 2-point function of e.m. tensors in arbitrary dimension $d$ in coordinate representation is given by

$$
\left\langle T_{\mu \nu}(x) T_{\rho \sigma}(0)\right\rangle=\frac{c / 2}{x^{2 d}}\left(I_{\mu \rho}(x) I_{\nu \sigma}(x)+I_{\nu \rho}(x) I_{\mu \sigma}(x)-\frac{2}{d} \eta_{\mu \nu} \eta_{\rho \sigma}\right)
$$

where

$$
I_{\mu \nu}(x)=\eta_{\mu \nu}-2 \frac{x_{\mu} x_{\nu}}{x^{2}} .
$$

As before, it can be regularized by writing down a differential operator which, acting on an integrable function, generates it for $x \neq 0$. One possibility for $d \geq 3$ is the following ${ }^{5}$

$$
\begin{aligned}
\left\langle T_{\mu \nu}(x) T_{\rho \sigma}(0)\right\rangle= & -\frac{c / 2}{2(d-2)^{2} d\left(d^{2}-1\right)} \mathcal{D}_{\mu \nu \rho \sigma}^{(1)}\left(\frac{1}{x^{2 d-4}}\right) \\
& +\frac{c / 2}{2(d-2)^{2} d(d+1)} \mathcal{D}_{\mu \nu \rho \sigma}^{(2)}\left(\frac{1}{x^{2 d-4}}\right),
\end{aligned}
$$

\footnotetext{
${ }^{5}$ Notice that for $d>4$, the function $1 / x^{2 d-4}$ is indeed integrable, while we have a function which is $\log$ divergent for $d=4$ and linearly divergent for $d=3$ and in both cases we need a regularization. In the spirit of differential regularization, we may use the following identities

$$
\begin{aligned}
& d=3: \quad \frac{1}{x^{2}}=\frac{1}{2} \square \log \mu^{2} x^{2}, \\
& d=4: \quad \frac{1}{x^{4}}=-\frac{1}{4} \square \frac{\log \mu^{2} x^{2}}{x^{2}},
\end{aligned}
$$

where $\log \mu^{2} x^{2}$ and $\left(\log \mu^{2} x^{2}\right) / x^{2}$ are integrable functions in the respective dimension.
} 
where

$$
\begin{aligned}
\mathcal{D}_{\mu \nu \rho \sigma}^{(1)}= & \partial_{\mu} \partial_{\nu} \partial_{\rho} \partial_{\sigma}-\left(\eta_{\mu \nu} \partial_{\rho} \partial_{\sigma}+\eta_{\rho \sigma} \partial_{\mu} \partial_{\nu}\right) \square+\eta_{\mu \nu} \eta_{\rho \sigma} \square \square, \\
\mathcal{D}_{\mu \nu \rho \sigma}^{(2)}= & \partial_{\mu} \partial_{\nu} \partial_{\rho} \partial_{\sigma}-\frac{1}{2}\left(\eta_{\mu \rho} \partial_{\nu} \partial_{\sigma}+\eta_{\nu \rho} \partial_{\mu} \partial_{\sigma}+\eta_{\mu \sigma} \partial_{\nu} \partial_{\rho}+\eta_{\nu \sigma} \partial_{\mu} \partial_{\rho}\right) \square \\
& +\frac{1}{2}\left(\eta_{\mu \rho} \eta_{\nu \sigma}+\eta_{\nu \rho} \eta_{\mu \sigma}\right) \square \square .
\end{aligned}
$$

Both these operators are conserved but not traceless:

$$
\begin{aligned}
& \eta^{\mu \nu} \mathcal{D}_{\mu \nu \rho \sigma}^{(1)}=-(d-1)\left(\partial_{\rho} \partial_{\sigma}-\eta_{\rho \sigma} \square\right) \square, \\
& \eta^{\mu \nu} \mathcal{D}_{\mu \nu \rho \sigma}^{(2)}=-\left(\partial_{\rho} \partial_{\sigma}-\eta_{\rho \sigma} \square\right) \square,
\end{aligned}
$$

nonetheless (5.3) is both conserved and traceless. The expression (5.3) coincides with (5.1) for $x \neq 0$, it is conserved and traceless.

There are, as usual, ambiguities in the definitions of the operators (5.4) and (5.5) for $x=0$. Particularly, in $d=4$ we may consider the most general modification that one could add to the expression (5.3), namely

$$
\begin{aligned}
\mathcal{A}_{\mu \nu \rho \sigma}= & {\left[A \partial_{\mu} \partial_{\nu} \partial_{\rho} \partial_{\sigma} \square+B\left(\eta_{\mu \rho} \partial_{\nu} \partial_{\sigma}+\eta_{\nu \rho} \partial_{\mu} \partial_{\sigma}+\eta_{\mu \sigma} \partial_{\nu} \partial_{\rho}+\eta_{\nu \sigma} \partial_{\mu} \partial_{\rho}\right) \square^{2}\right.} \\
& \left.+C\left(\eta_{\mu \nu} \partial_{\rho} \partial_{\sigma}+\eta_{\rho \sigma} \partial_{\mu} \partial_{\nu}\right) \square^{2}+D\left(\eta_{\mu \rho} \eta_{\nu \sigma}+\eta_{\nu \rho} \eta_{\mu \sigma}\right) \square^{3}+E \eta_{\mu \nu} \eta_{\rho \sigma} \square^{3}\right] \frac{1}{x^{2}} .
\end{aligned}
$$

Conservation of $\mathcal{A}$ requires

$$
C=-A+2 D, \quad D=-B, \quad E=A+2 B .
$$

With these conditions the trace of $\mathcal{A}$ is

$$
\mathcal{A}_{\mu \rho \sigma}^{\mu}=-4 \pi^{2}(3 A+4 B)\left(\eta_{\rho \sigma} \square-\partial_{\rho} \partial_{\sigma}\right) \square \delta(x) .
$$

This corresponds to the trivial anomaly $\square R$, which can be subtracted away by adding a local Weyl invariant counterterm to the action. The existence of a definition of our differential operators which do not imply in the existence of this anomaly reflects the fact that it is a trivial anomaly.

\subsection{2-point correlator with Feynman diagrams}

The computation is very similar to the one in $2 d$. Again, the only diagram that contributes is the one of figure 1 and we have ${ }^{6}$

$$
\left\langle T_{\mu \nu}(x) T_{\lambda \rho}(y)\right\rangle=4 \int \frac{d^{4} k}{(2 \pi)^{4}} e^{-i k(x-y)} \tilde{\mathcal{T}}_{\mu \nu \lambda \rho}(k)
$$

where

$$
\tilde{\mathcal{T}}_{\mu \nu \lambda \rho}(k)=-\frac{1}{64} \int \frac{d^{4} p}{(2 \pi)^{4}} \operatorname{tr}\left(\frac{1}{\not p}(2 p-k)_{\mu} \gamma_{\nu} \frac{1}{\not p-\not k}(2 p-k)_{\lambda} \gamma_{\rho} \frac{1+\gamma_{5}}{2}\right)+\left\{\begin{array}{l}
\mu \leftrightarrow \nu \\
\lambda \leftrightarrow \rho
\end{array}\right\}
$$

\footnotetext{
${ }^{6}$ For the factor of 4 in (5.11), see the footnote in section 4.
} 
To evaluate it we use dimensional regularization. After introducing the Feynman parameter $x$ and shifting $p$ as follows: $p \rightarrow p-(1-x) k,(5.12)$ writes $^{7}$

$$
\begin{aligned}
\tilde{\mathcal{T}}_{\mu \nu \lambda \rho}(k)= & -\frac{1}{32} \int_{0}^{1} d x \int \frac{d^{4} p}{(2 \pi)^{4}} \int \frac{d^{\delta} \ell}{(2 \pi)^{\delta}} \frac{(2 p+(1-2 x) k)_{\mu}(2 p+(1-2 x) k)_{\lambda}}{\left(p^{2}+x(1-x) k^{2}-\ell^{2}\right)^{2}} \\
& \times\left[(p+(1-x) k)^{\sigma}(p-x k)^{\tau}\left(\eta_{\sigma \nu} \eta_{\tau \rho}-\eta_{\sigma \tau} \eta_{\nu \rho}+\eta_{\sigma \rho} \eta_{\nu \tau}-i \epsilon_{\sigma \nu \tau \rho}\right)-\ell^{2} \eta_{\nu \rho}\right]
\end{aligned}
$$

After the integrations (first $\ell$, then $p$, then $x$ ) one finds ${ }^{8}$

$$
\tilde{\mathcal{T}}_{\mu \nu \lambda \rho}(k)=\tilde{\mathcal{D}}_{\mu \nu \lambda \rho}(k)+\tilde{\mathcal{F}}_{\mu \nu \lambda \rho}(k)+\tilde{\mathcal{L}}_{\mu \nu \lambda \rho}(k)
$$

where

$$
\begin{aligned}
\tilde{\mathcal{D}}_{\mu \nu \lambda \rho}(k)= & -\frac{i}{32(4 \pi)^{2}} \frac{1}{15 \delta}\left[8 k_{\mu} k_{\nu} k_{\lambda} k_{\rho}+4 k^{2}\left(k_{\mu} k_{\nu} \eta_{\lambda \rho}+k_{\lambda} k_{\rho} \eta_{\mu \nu}\right)\right. \\
& -6 k^{2}\left(k_{\mu} k_{\lambda} \eta_{\nu \rho}+k_{\nu} k_{\lambda} \eta_{\mu \rho}+k_{\mu} k_{\rho} \eta_{\nu \lambda}+k_{\nu} k_{\rho} \eta_{\mu \lambda}\right) \\
& \left.-4 k^{4} \eta_{\mu \nu} \eta_{\lambda \rho}+6 k^{4}\left(\eta_{\mu \lambda} \eta_{\nu \rho}+\eta_{\mu \rho} \eta_{\nu \lambda}\right)\right]
\end{aligned}
$$

which is divergent for $\delta \rightarrow 0$, but conserved and traceless,

$$
\begin{aligned}
\tilde{\mathcal{L}}_{\mu \nu \lambda \rho}(k)= & -\frac{i}{32(4 \pi)^{2}} \frac{\log k^{2}}{30}\left[8 k_{\mu} k_{\nu} k_{\lambda} k_{\rho}+4 k^{2}\left(k_{\mu} k_{\nu} \eta_{\lambda \rho}+k_{\lambda} k_{\rho} \eta_{\mu \nu}\right)\right. \\
& -6 k^{2}\left(k_{\mu} k_{\lambda} \eta_{\nu \rho}+k_{\nu} k_{\lambda} \eta_{\mu \rho}+k_{\mu} k_{\rho} \eta_{\nu \lambda}+k_{\nu} k_{\rho} \eta_{\mu \lambda}\right) \\
& \left.-4 k^{4} \eta_{\mu \nu} \eta_{\lambda \rho}+6 k^{4}\left(\eta_{\mu \lambda} \eta_{\nu \rho}+\eta_{\mu \rho} \eta_{\nu \lambda}\right)\right]
\end{aligned}
$$

which is also conserved and traceless, and

$$
\begin{aligned}
\tilde{\mathcal{F}}_{\mu \nu \lambda \rho}(k)= & -\frac{i}{32(4 \pi)^{2}} \frac{1}{30}\left[8\left(\gamma-\log 4 \pi+\frac{31}{450}\right) k_{\mu} k_{\nu} k_{\lambda} k_{\rho}\right. \\
& +2\left(1-\gamma+\log 4 \pi+\frac{31}{150}\right) k^{2}\left(k_{\mu} k_{\lambda} \eta_{\nu \rho}+k_{\nu} k_{\lambda} \eta_{\mu \rho}+k_{\mu} k_{\rho} \eta_{\nu \lambda}+k_{\nu} k_{\rho} \eta_{\mu \lambda}\right) \\
& +k^{4}\left(\frac{10}{3}-4 \gamma+4 \log 4 \pi-\frac{47}{225}\right) \eta_{\mu \nu} \eta_{\lambda \rho} \\
& -k^{4}\left(\frac{17}{3}-6 \gamma+6 \log 4 \pi\right)\left(\eta_{\mu \lambda} \eta_{\nu \rho}+\eta_{\mu \rho} \eta_{\nu \lambda}\right) \\
& \left.-k^{2}\left(4-4 \gamma+4 \log 4 \pi+\frac{47}{450}\right)\left(k_{\mu} k_{\nu} \eta_{\lambda \rho}+k_{\lambda} k_{\rho} \eta_{\mu \nu}\right)\right]
\end{aligned}
$$

which is neither conserved nor traceless.

Let us consider first $\tilde{\mathcal{L}}$. We recall the Fourier transform

$$
\int d^{4} x e^{i k x} \frac{1}{x^{2}} \log \mu^{2} x^{2}=\frac{4 \pi^{2} i}{k^{2}}\left(\log 2-\gamma-\log \frac{k^{2}}{\mu^{2}}\right) .
$$

Therefore, up to the term proportional to $(\log 2-\gamma)$, by Fourier transforming (5.3) we obtain precisely (5.16) with $c=1 / \pi^{4}$, in agreement with the results of $[3,4]$. The term

\footnotetext{
${ }^{7}$ We use the mostly minus signature for the metric.

${ }^{8}$ To do integration properly we have to Wick rotate the momenta and, after integration rotate them back to the Lorentzian signature. We understand this here.
} 
proportional to $(\log 2-\gamma)$ is to be added to (5.17). Now the divergence of $\tilde{\mathcal{T}}$ contains three independent terms proportional to $k^{2} k_{\nu} k_{\lambda} k_{\rho}, k^{4} k_{\nu} \eta_{\lambda \rho}$ and $k^{4}\left(k_{\lambda} \eta_{\nu \rho}+k_{\rho} \eta_{\nu \lambda}\right)$, respectively, while the trace contains two independent terms proportional to $k^{2} k_{\lambda} k_{\rho}$ and $k^{4} \eta_{\lambda \rho}$. On the other hand the ambiguity (5.8) contains the same 5 independent terms with arbitrary coefficients. Therefore it is always possible to set to zero both the divergence and the trace of $\tilde{\mathcal{T}}$ by subtracting suitable counterterms. In the same way one can argue with the divergent term $\tilde{\mathcal{D}}$. This term deserves a comment: it is traceless and divergenceless, but it is infinite, so it must be subtracted away along with the $\tilde{\mathcal{F}}$ term. Both $\mathcal{F}$ and $\mathcal{D}$, the Fourier anti-transforms of $\tilde{\mathcal{F}}$ and $\tilde{\mathcal{D}}$, are contact terms and they can be written in a compact form as

$$
\begin{aligned}
\left\langle\left\langle T_{\mu \nu}(x)\right\rangle\right\rangle= & A^{\prime} \partial_{\mu} \partial_{\nu} \partial_{\lambda} \partial_{\rho} h^{\lambda \rho}(x)+B^{\prime}\left(\square \partial_{\mu} \partial_{\lambda} h_{\nu}^{\lambda}(x)+\square \partial_{\nu} \partial_{\lambda} h_{\mu}^{\lambda}(x)\right)+C^{\prime} \eta_{\mu \nu} \square^{2} h(x) \\
& +D^{\prime} \square^{2} h_{\mu \nu}(x)+E^{\prime}\left(\square \partial_{\mu} \partial_{\nu} h(x)+\eta_{\mu \nu} \square \partial_{\lambda} \partial_{\rho} h^{\lambda \rho}(x)\right)
\end{aligned}
$$

where $h=h_{\lambda}^{\lambda}$ and $A^{\prime}, B^{\prime}, C^{\prime}, D^{\prime}, E^{\prime}$ are numerical coefficients that contain also a part $\sim \frac{1}{\delta}$. The local term to be subtracted from the action is proportional to

$$
\begin{gathered}
\int d^{4} x\left(\frac{A^{\prime}}{2} h^{\mu \nu} \partial_{\mu} \partial_{\nu} \partial_{\lambda} \partial_{\rho} h^{\lambda \rho}+B^{\prime} h^{\mu \nu} \square \partial_{\mu} \partial_{\lambda} h_{\nu}^{\lambda}\right. \\
\left.+\frac{C^{\prime}}{2} h \square^{2} h+\frac{D^{\prime}}{2} h^{\mu \nu} \square^{2} h_{\mu \nu}+E^{\prime} h^{\mu \nu} \square \partial_{\mu} \partial_{\nu} h\right)
\end{gathered}
$$

We can conclude that the (regularized) Feynman diagram approach to the 2-point correlator is equivalent to regularizing the 2-point function calculated with the Wick theorem approach. But we can draw also another, less pleasant, conclusion. Like in $2 d$, the Feynman diagrams coupled to dimensional regularization may also produce unwelcome terms, such as the $\mathcal{D}$ and $\mathcal{F}$ terms above, which must be subtracted away by hand.

Finally we notice that, once (5.20) has been subtracted away, not only the nonvanishing trace and divergence of the em tensor disappear, but the full contact term (5.19) gets canceled. Thus the regularized 2-point correlator of the e.m. tensor coincides with the "bare" expression.

\section{The 3-point correlator}

The calculation of the 3-point correlator brings new elements into the game. First and foremost new (nontrivial) anomalies, but also an enormous complexity as compared to the 2-point correlator. In this section we first show that generically at non-coincident points the 3 -point function of e.m. tensors in $4 d$ does not possess a parity-odd contribution due to the permutation symmetry of the correlator. Then we compute the "bare" 3-point correlator by means of the Wick theorem in the same specific chiral fermionic model considered above, disregarding regularization. We find that, as expected, the parity-odd part identically vanishes. Subsequently we compute the same amplitude using Feynman diagrams and regularize it. It turns out that not only the parity-even but also the parity-odd trace of the e.m. tensor is nonvanishing. We will explain this apparent paradox in section 7 . 


\subsection{No-go for parity-odd contributions}

In this subsection we will review the fact that in four dimensions there are no parityodd "bare" contributions to the 3-point function of energy-momentum tensors, which has already been emphasized in $[7,8]$.

A very powerful tool to analyse which tensorial structures can exist in a given correlation function in a CFT is the embedding formalism as it was formulated in [6]. In their language, to construct conformally covariant tensorial structures becomes a game of putting together building blocks respecting the tensorial requirements of your correlator. Particularly for the 3-point function of e.m. tensors we have seven building blocks. These building blocks are written in terms of points $P_{i}$ of the six-dimensional embedding space and lightlike polarization vectors $Z_{i}$. Three of them depend on two points, namely

$$
\begin{aligned}
& H_{12}=-2\left[\left(Z_{1} \cdot Z_{2}\right)\left(P_{1} \cdot P_{2}\right)-\left(Z_{1} \cdot P_{2}\right)\left(Z_{2} \cdot P_{1}\right)\right], \\
& H_{23}=-2\left[\left(Z_{2} \cdot Z_{3}\right)\left(P_{2} \cdot P_{3}\right)-\left(Z_{2} \cdot P_{3}\right)\left(Z_{3} \cdot P_{2}\right)\right], \\
& H_{13}=-2\left[\left(Z_{1} \cdot Z_{3}\right)\left(P_{1} \cdot P_{3}\right)-\left(Z_{1} \cdot P_{3}\right)\left(Z_{3} \cdot P_{1}\right)\right] .
\end{aligned}
$$

Four of them depend on three points, three being parity-even, namely

$$
\begin{aligned}
& V_{1}=\frac{\left(Z_{1} \cdot P_{2}\right)\left(P_{1} \cdot P_{3}\right)-\left(Z_{1} \cdot P_{3}\right)\left(P_{1} \cdot P_{2}\right)}{P_{2} \cdot P_{3}}, \\
& V_{2}=\frac{\left(Z_{2} \cdot P_{3}\right)\left(P_{2} \cdot P_{1}\right)-\left(Z_{2} \cdot P_{1}\right)\left(P_{2} \cdot P_{3}\right)}{P_{3} \cdot P_{1}}, \\
& V_{3}=\frac{\left(Z_{3} \cdot P_{1}\right)\left(P_{3} \cdot P_{2}\right)-\left(Z_{3} \cdot P_{2}\right)\left(P_{3} \cdot P_{1}\right)}{P_{1} \cdot P_{2}},
\end{aligned}
$$

while the last one is parity-odd, being the only object that one may construct with an epsilon tensor, i.e.

$$
O_{123}=\epsilon\left(Z_{1}, Z_{2}, Z_{3}, P_{1}, P_{2}, P_{3}\right)
$$

Our job now is to put together these objects to form a conformally covariant object with the tensorial structure of the 3-point function of e.m. tensors. Particularly, the objects that we will construct must present twice each polarization vector $Z_{i}$, since each $Z_{i}$ is associated with one index of the $i$-th e.m. tensor. Since we are interested on parity-odd terms we will necessarily have the building block $O_{123}$ which already takes care of one factor of each $Z_{i}$, thus it is clear that our only options are

$$
\begin{aligned}
& T_{1}=O_{123} V_{1} V_{2} V_{3}, \\
& T_{2}=O_{123}\left(V_{1} H_{23}+V_{2} H_{13}+V_{3} H_{12}\right) .
\end{aligned}
$$

In the following we will show that both $T_{1}$ and $T_{2}$ are antisymmetric under the permutation of 1 and 2 for example, which forbids them to be present in the 3-point function of e.m. tensors. By inspection of the expressions (6.1)-(6.7) we see that under the exchange 
of 1 and 2 our building blocks change as follows:

$$
\begin{aligned}
H_{12} & \rightarrow H_{12}, \\
H_{23} & \rightarrow H_{13}, \\
H_{13} & \rightarrow H_{23}, \\
V_{1} & \rightarrow-V_{2}, \\
V_{2} & \rightarrow-V_{1}, \\
V_{3} & \rightarrow-V_{3}, \\
O_{123} & \rightarrow O_{123} .
\end{aligned}
$$

From these rules it is clear that both $T_{1}$ and $T_{2}$ are antisymmetric under the exchange of 1 and 2 . Of course the same result holds for the exchanges $1 \leftrightarrow 3$ and $2 \leftrightarrow 3$.

\subsection{The "bare" parity-odd 3-point correlator}

Consider a free chiral fermion $\psi_{L}$ in four dimensions which has the 2-point function ${ }^{9}$

$$
\left\langle\psi_{L}(x) \overline{\psi_{L}}(y)\right\rangle=\frac{i}{2 \pi^{2}} \frac{\gamma \cdot(x-y)}{(x-y)^{4}} P_{L}, \quad P_{L}=\frac{1-\gamma_{5}}{2},
$$

and the e.m. tensor

$$
T_{\mu \nu}=\frac{i}{4}\left(\overline{\psi_{L}} \gamma_{\mu} \stackrel{\leftrightarrow}{\partial}_{\nu} \psi_{L}+\mu \leftrightarrow \nu\right), \text { where } \stackrel{\leftrightarrow}{\partial}_{\nu} \equiv \partial_{\nu}-\overleftarrow{\partial}_{\nu}
$$

Since we are dealing with a free theory we are able to compute the 3-point function of e.m. tensors by applying the Wick theorem. Using the explicit form of the e.m. tensor (6.11) we write

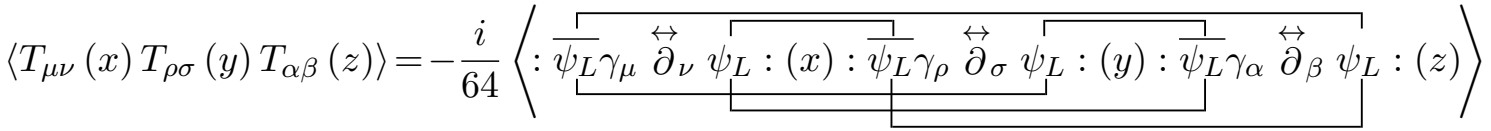

$$
\begin{aligned}
& + \text { symmetrization. }
\end{aligned}
$$

There are two ways to fully contract these fields, as shown in equation (6.12). Each of the contractions is composed by a certain tensor with six indices $f_{\nu a \sigma b \beta c}^{(i)}$ contracted with a trace of six gamma matrices and a projector $P_{L}$, namely

$$
f_{\nu a \sigma b \beta c}^{(1)} \operatorname{tr}\left(\gamma_{\mu} \gamma^{a} \gamma_{\rho} \gamma^{b} \gamma_{\alpha} \gamma^{c} P_{L}\right) \text { and } f_{\nu a \sigma b \beta c}^{(2)} \operatorname{tr}\left(\gamma_{\mu} \gamma^{a} \gamma_{\alpha} \gamma^{b} \gamma_{\rho} \gamma^{c} P_{L}\right)
$$

where the upper index of $f$ is 1 for the first way of contracting and 2 for the second way. The ordering of the free indices in the trace are given by the two ways of performing the full contraction. The functions $f_{\nu a \sigma b \beta c}^{(i)}$ are composed by eight terms which are the eight forms of distributing the derivatives in the right hand side of (6.12). We will show that in

\footnotetext{
${ }^{9}$ The factor of $\frac{1}{2 \pi^{2}}$ in the propagator of a fermion in $4 d$ is needed in order for its Fourier-transform to give the usual propagator, namely $\frac{i}{\not p}$.
} 
reality $f^{(1)}$ and $f^{(2)}$ are the same object. To see this we will only need to exchange $a$ with $c$ in the expression for the second way of contracting, i.e.

$$
f_{\nu a \sigma b \beta c}^{(2)} \operatorname{tr}\left(\gamma_{\mu} \gamma^{a} \gamma_{\alpha} \gamma^{b} \gamma_{\rho} \gamma^{c} P_{L}\right)=f_{\nu a \sigma b \beta c}^{(1)} \operatorname{tr}\left(\gamma_{\mu} \gamma^{c} \gamma_{\alpha} \gamma^{b} \gamma_{\rho} \gamma^{a} P_{L}\right)
$$

Hence, the sum of the two ways of contracting will simplify to

$$
f_{\nu a \sigma b \beta c}^{(1)}\left[\operatorname{tr}\left(\gamma_{\mu} \gamma^{a} \gamma_{\rho} \gamma^{b} \gamma_{\alpha} \gamma^{c} P_{L}\right)+\operatorname{tr}\left(\gamma_{\mu} \gamma^{c} \gamma_{\alpha} \gamma^{b} \gamma_{\rho} \gamma^{a} P_{L}\right)\right] .
$$

It is possible to put the second trace in the form $\operatorname{tr}\left(\gamma_{\rho} \gamma^{a} \gamma_{\mu} \gamma^{c} \gamma_{\alpha} \gamma^{b} P_{L}\right)$, which reduces our final expression to

$$
f_{\nu a \sigma b \beta c}^{(1)}\left[\operatorname{tr}\left(\gamma_{\mu} \gamma^{a} \gamma_{\rho} \gamma^{b} \gamma_{\alpha} \gamma^{c} P_{L}\right)+\operatorname{tr}\left(\gamma_{\rho} \gamma^{a} \gamma_{\mu} \gamma^{c} \gamma_{\alpha} \gamma^{b} P_{L}\right)\right] .
$$

The trace of six gamma matrices and a gamma five is given by

$$
\begin{aligned}
\operatorname{tr}\left(\gamma_{\mu} \gamma_{a} \gamma_{\rho} \gamma_{b} \gamma_{\alpha} \gamma_{c} \gamma_{5}\right)= & 4 i\left(\eta_{\mu a} \epsilon_{\rho b \alpha c}-\eta_{\mu \rho} \epsilon_{a b \alpha c}+\eta_{\rho a} \epsilon_{\mu b \alpha c}\right. \\
& \left.+\eta_{\alpha c} \epsilon_{\mu a \rho b}-\eta_{b c} \epsilon_{\mu a \rho \alpha}+\eta_{\alpha b} \epsilon_{\mu a \rho c}\right)
\end{aligned}
$$

As one can easily check, the trace (6.17) is antisymmetric under the exchange ( $\mu \leftrightarrow \rho, b \leftrightarrow c$ ), thus the odd part of the correlation function is zero.

Now we will work out what are the functions $f^{(i)}$ and show the relation between $f^{(1)}$ and $f^{(2)}$ mentioned above. From the first way of contracting we derive the expression

$$
\operatorname{tr}\left[\gamma_{\mu} \partial_{\nu}\left(\gamma^{a} \partial_{a} \frac{1}{(x-y)^{2}} P_{L}\right) \gamma_{\rho} \partial_{\sigma}\left(\gamma^{b} \partial_{b} \frac{1}{(y-z)^{2}} P_{L}\right) \gamma_{\alpha} \partial_{\beta}\left(\gamma^{c} \partial_{c} \frac{1}{(z-x)^{2}} P_{L}\right)\right]+\cdots,
$$

where the ellipsis stand for the seven other ways of organizing the derivatives $\partial_{\nu}, \partial_{\sigma}$ and $\partial_{\beta}$. From (6.18) we see that we will have some expression that we call $f^{(1)}$ contracted with $\operatorname{tr}\left(\gamma_{\mu} \gamma^{a} \gamma_{\rho} \gamma^{b} \gamma_{\alpha} \gamma^{c} P_{L}\right)$. The expression for $f^{(1)}$ can be read off from (6.18):

$$
\begin{aligned}
f_{\nu a \sigma b \beta c}^{(1)}= & \partial_{\nu}^{x} \partial_{a}^{x} \frac{1}{(x-y)^{2}} \partial_{\sigma}^{y} \partial_{b}^{y} \frac{1}{(y-z)^{2}} \partial_{\beta}^{z} \partial_{c}^{z} \frac{1}{(z-x)^{2}}-\partial_{\sigma}^{y} \partial_{a}^{x} \frac{1}{(x-y)^{2}} \partial_{\beta}^{z} \partial_{b}^{y} \frac{1}{(y-z)^{2}} \partial_{\nu}^{x} \partial_{c}^{z} \frac{1}{(z-x)^{2}} \\
& -\partial_{\sigma}^{y} \partial_{\nu}^{x} \partial_{a}^{x} \frac{1}{(x-y)^{2}}\left[\partial_{b}^{y} \frac{1}{(y-z)^{2}} \partial_{\beta}^{z} \partial_{c}^{z} \frac{1}{(z-x)^{2}}-\partial_{\beta}^{z} \partial_{b}^{y} \frac{1}{(y-z)^{2}} \partial_{c}^{z} \frac{1}{(z-x)^{2}}\right] \\
& -\partial_{\beta}^{z} \partial_{\sigma}^{y} \partial_{b}^{y} \frac{1}{(y-z)^{2}}\left[\partial_{\nu}^{x} \partial_{a}^{x} \frac{1}{(x-y)^{2}} \partial_{c}^{z} \frac{1}{(z-x)^{2}}-\partial_{a}^{x} \frac{1}{(x-y)^{2}} \partial_{\nu}^{x} \partial_{c}^{z} \frac{1}{(z-x)^{2}}\right] \\
& -\partial_{\nu}^{x} \partial_{\beta}^{z} \partial_{c}^{z} \frac{1}{(z-x)^{2}}\left[\partial_{a}^{x} \frac{1}{(x-y)^{2}} \partial_{\sigma}^{y} \partial_{b}^{y} \frac{1}{(y-z)^{2}}-\partial_{\sigma}^{y} \partial_{a}^{x} \frac{1}{(x-y)^{2}} \partial_{b}^{y} \frac{1}{(y-z)^{2}}\right] .
\end{aligned}
$$

The second way of contracting give us the expression

$$
\operatorname{tr}\left[\gamma_{\mu} \partial_{\nu}\left(\gamma^{a} \partial_{a} \frac{1}{(x-z)^{2}} P_{L}\right) \gamma_{\alpha} \partial_{\beta}\left(\gamma^{b} \partial_{b} \frac{1}{(z-y)^{2}} P_{L}\right) \gamma_{\alpha} \partial_{\beta}\left(\gamma^{c} \partial_{c} \frac{1}{(y-x)^{2}} P_{L}\right)\right]+\cdots,
$$


from where we may read off the expression for $f^{(2)}$ :

$$
\begin{aligned}
f_{\nu a \sigma b \beta c}^{(2)}= & \partial_{\nu}^{x} \partial_{a}^{x} \frac{1}{(x-z)^{2}} \partial_{\beta}^{z} \partial_{b}^{z} \frac{1}{(z-y)^{2}} \partial_{\sigma}^{y} \partial_{c}^{y} \frac{1}{(y-x)^{2}}-\partial_{\beta}^{z} \partial_{a}^{x} \frac{1}{(x-z)^{2}} \partial_{\sigma}^{y} \partial_{b}^{z} \frac{1}{(z-y)^{2}} \partial_{\nu}^{x} \partial_{c}^{y} \frac{1}{(y-x)^{2}} \\
& -\partial_{\nu}^{x} \partial_{\sigma}^{y} \partial_{c}^{y} \frac{1}{(y-x)^{2}}\left[\partial_{a}^{x} \frac{1}{(x-z)^{2}} \partial_{\beta}^{z} \partial_{b}^{z} \frac{1}{(z-y)^{2}}-\partial_{\beta}^{z} \partial_{a}^{x} \frac{1}{(x-z)^{2}} \partial_{b}^{z} \frac{1}{(z-y)^{2}}\right] \\
& -\partial_{\sigma}^{y} \partial_{\beta}^{z} \partial_{b}^{z} \frac{1}{(z-y)^{2}}\left[\partial_{\nu}^{x} \partial_{a}^{x} \frac{1}{(x-z)^{2}} \partial_{c}^{y} \frac{1}{(y-x)^{2}}-\partial_{a}^{x} \frac{1}{(x-z)^{2}} \partial_{\nu}^{x} \partial_{c}^{y} \frac{1}{(y-x)^{2}}\right] \\
& -\partial_{\beta}^{z} \partial_{\nu}^{x} \partial_{a}^{x} \frac{1}{(x-z)^{2}}\left[\partial_{b}^{z} \frac{1}{(z-y)^{2}} \partial_{\sigma}^{y} \partial_{c}^{y} \frac{1}{(y-x)^{2}}-\partial_{\sigma}^{y} \partial_{b}^{z} \frac{1}{(z-y)^{2}} \partial_{c}^{y} \frac{1}{(y-x)^{2}}\right]
\end{aligned}
$$

It is now a straightforward exercise to check that if one exchanges $a$ with $c$ in the expression of $f_{\nu a \sigma b \beta c}^{(2)}$ one gets $f_{\nu a \sigma b \beta c}^{(1)}$, i.e.

$$
f_{\nu c \sigma b \beta a}^{(2)}=f_{\nu a \sigma b \beta c}^{(1)} .
$$

We remind the reader that in this computation we have ignored coincident point singularities. The next task will be to take them into account, which will be done in momentu space.

\subsection{Relevant Fourier transforms}

In the next subsection, in order to compute the 3-point amplitude of the e.m. tensor, with the Feynman diagram technique we will use (momentum space) Feynman diagrams. Although essentially equivalent to the Wick theorem they lend themselves more naturally to regularization. The two techniques are related by Fourier transform. Hereby we collect a series of Fourier transforms of distributions that are used in our calculations. The source is [28]. The notation is as follows

$$
\mathcal{F}[\phi(x)](k) \equiv \tilde{\phi}(k)=\int d^{4} x e^{i k x} \phi(x), \quad \phi(x)=\int \frac{d^{4} k}{(2 \pi)^{4}} e^{-i k x} \tilde{\phi}(k)
$$

In particular

$$
\begin{aligned}
\int d^{4} x e^{i k x} \frac{1}{x^{2}} & =\frac{4 \pi^{2} i}{k^{2}} \\
\int d^{4} x e^{i k x} \frac{\log x^{2} \mu^{2}}{x^{2}} & =-\frac{4 \pi^{2} i}{k^{2}} \log \left(\frac{-k^{2}}{\bar{\mu}^{2}}\right),
\end{aligned}
$$

where $\bar{\mu}^{2} \equiv 2 \mu^{2} e^{-\gamma}, \gamma=0.57721 \ldots$ being the Euler constant. As we have seen this is essentially what one needs to compute the Fourier transform of the 2-point correlator. The novel feature in the calculation of the 3-point correlator is the appearance of products of similar expressions in different points, a prototype being

$$
\frac{1}{(x-y)^{2}(x-z)^{2}(y-z)^{2}} \text {. }
$$


This is singular at coincident points and has a non-integrable singularity at $x=y=z=0$. Ignoring this let us proceed to Fourier-transforming it

$$
\begin{aligned}
\int d^{4} x d^{4} y d^{4} z \frac{e^{i\left(k_{1} x+k_{2} y-q z\right)}}{(x-y)^{2}(x-z)^{2}(y-z)^{2}} & =\int d^{4} x d^{4} y d^{4} z \frac{e^{i\left(k_{1} x+k_{2} y+\left(k_{1}+k_{2}-q\right) z\right)}}{(x-y)^{2} x^{2} y^{2}} \\
& =(2 \pi)^{4} \delta\left(q-k_{1}-k_{2}\right) \int d^{4} x d^{4} y \frac{e^{i\left(k_{1} x+k_{2} y\right)}}{(x-y)^{2} x^{2} y^{2}}
\end{aligned}
$$

Let us set $f(x, y)=\frac{1}{(x-y)^{2} x^{2} y^{2}}$. Then, using the convolution theorem, the Fourier transform of $f$ with respect to $x$ is

$$
\begin{aligned}
\mathcal{F}_{x}[f(x, y)]\left(k_{1}\right) & =\int d^{4} x e^{i k_{1} x} f(x, y)=\frac{1}{y^{2}} \int d^{4} x \frac{e^{i k_{1} x}}{x^{2}(x-y)^{2}} \\
& =\frac{1}{y^{2}} \int \frac{d^{4} p}{(2 \pi)^{4}} \mathcal{F}_{x}\left[\frac{1}{x^{2}}\right]\left(k_{1}-p\right) \mathcal{F}_{x}\left[\frac{1}{(x-y)^{2}}\right](p) \\
& =-\frac{1}{y^{2}} \int d^{4} p \frac{e^{i p y}}{p^{2}\left(p-k_{1}\right)^{2}} .
\end{aligned}
$$

Therefore

$$
\begin{aligned}
\int d^{4} x d^{4} y \frac{e^{i\left(k_{1} x+k_{2} y\right)}}{(x-y)^{2} x^{2} y^{2}} & =\int d^{4} y e^{i k_{2} y} \mathcal{F}_{x}[f(x, y)]\left(k_{1}\right) \\
& =-i(2 \pi)^{6} \int \frac{d^{4} p}{(2 \pi)^{4}} \frac{1}{p^{2}\left(p-k_{1}\right)^{2}\left(p+k_{2}\right)^{2}} .
\end{aligned}
$$

We can now compute the r.h.s. of (6.28) in the usual way by introducing a Feynman parametrization in terms of two parameters $u, v$ :

$$
\int \frac{d^{4} p}{(2 \pi)^{4}} \frac{1}{p^{2}\left(p-k_{1}\right)^{2}\left(p+k_{2}\right)^{2}}=\int_{0}^{1} d u \int_{0}^{1-u} d v \int \frac{d^{4} p^{\prime}}{(2 \pi)^{4}} \frac{1}{\left(p^{\prime 2}-\ell^{2}+\Delta\right)^{3}}
$$

where $p^{\prime}=p-u k_{1}+v k_{2}$ and $\Delta=u(1-u) k_{1}^{2}+v(1-v) k_{2}^{2}+2 u v k_{1} k_{2}$. Performing the $p^{\prime}$ integral one gets

$$
\int_{0}^{1} d u \int_{0}^{1-u} d v \int \frac{d^{4} p}{(2 \pi)^{4}} \int \frac{d^{\delta} \ell}{(2 \pi)^{\delta}} \frac{1}{\left(p^{2}-\ell^{2}+\Delta\right)^{3}}=\frac{i}{2(4 \pi)^{2}} \int_{0}^{1} d u \int_{0}^{1-u} d v \frac{1}{\Delta}
$$

Our attitude will be to define the regularization of (6.25) as the Fourier anti-transform of the (6.30).

In general, however, the expressions we have to do with are not as simple as (6.30) and the integrals as simple as (6.28). The typical integral of the type (6.28) contains a polynomial of $p, k_{1}, k_{2}$ in the numerator of the integrand. In this case we have two ways to proceed: either we extend the running momentum $p$ to extra dimensions (dimensional regularization), as we have done in $2 \mathrm{~d}$, carry out the integration and Fourier-anti-transform the final result, or we reduce the calculations to a differential operator applied to the Fourier-anti-transform of (6.30) (differential regularization). Usually the former procedure is more convenient, while in many cases the latter is problematic.

Other analogous expressions are obtained in appendix D. 


\subsection{The parity-odd 3-point correlator with Feynman diagrams}

This section is devoted to the same calculation as in subsection (6.2), but with Feynman diagram techniques. In order to compute the 3-point function of the energy-momentum tensor for a chiral fermion, it is very convenient to couple it minimally to gravity and extract from the corresponding action the Feynman rules, as in $[22,30]$. The relevant formalism and notation is reviewed in appendix B. Due to the non polynomial character of the action the diagrams contributing to the trace anomaly are infinitely many. Fortunately, using diffeomorphism invariance, it is enough to determine the lowest order contributions and consistency takes care of the rest. There are two potential lowest order diagrams that may contribute. The first contribution, the bubble graph, turns out to give a vanishing contribution. The important term comes from the triangle graph. This has an incoming line with momentum $q=k_{1}+k_{2}$ with Lorentz indices $\mu, \nu$. The two outgoing lines have momenta $k_{1}, k_{2}$ with Lorentz labels $\lambda, \rho$ and $\alpha, \beta$, respectively. The contribution is formally written as

$$
\begin{aligned}
\mathcal{T}_{\mu \nu \alpha \beta \lambda \rho}^{(1)}\left(k_{1}, k_{2}\right)= & -\frac{1}{512} \int \frac{d^{4} p}{(2 \pi)^{4}} \operatorname{tr}\left[\left(\frac{1}{\not p}\left(\left(2 p-k_{1}\right)_{\lambda} \gamma_{\rho}+(\lambda \leftrightarrow \rho)\right) \frac{1}{\not p-\not k_{1}}\right.\right. \\
& \left.\left.\times\left(\left(2 p-2 k_{1}-k_{2}\right)_{\alpha} \gamma_{\beta}+(\alpha \leftrightarrow \beta)\right) \frac{1}{\not p-\not p}\left((2 p-q)_{\mu} \gamma_{\nu}+(\mu \leftrightarrow \nu)\right)\right) \frac{1+\gamma_{5}}{2}\right]
\end{aligned}
$$

to which the cross graph contribution $\mathcal{T}_{\mu \nu \alpha \beta \lambda \rho}^{(2)}\left(k_{1}, k_{2}\right)=\mathcal{T}_{\mu \nu \lambda \rho \alpha \beta}^{(1)}\left(k_{2}, k_{1}\right)$ has to be added. We regularize (6.31) as usual by introducing extra component of the momentum running around the loop $p \rightarrow p+\ell, \ell=\ell_{4}, \ldots, \ell_{\delta+4}$ :

$$
\begin{aligned}
\mathcal{T}_{\mu \nu \alpha \beta \lambda \rho}^{(1)}\left(k_{1}, k_{2}\right)= & -\frac{1}{512} \int \frac{d^{4} p}{(2 \pi)^{4}} \int \frac{d^{\delta} \ell}{(2 \pi)^{\delta}} \operatorname{tr}\left(\frac{\not p+\ell}{p^{2}-\ell^{2}}\left(2 p-k_{1}\right)_{\lambda} \gamma_{\rho}\right. \\
& \left.\times \frac{\not p+\ell-\not k_{1}}{\left(p-k_{1}\right)^{2}-\ell^{2}}\left(2 p-2 k_{1}-k_{2}\right)_{\alpha} \gamma_{\beta} \frac{\not p+\ell-\not l}{(p-q)^{2}-\ell^{2}}(2 p-q)_{\mu} \gamma_{\nu} \frac{1+\gamma_{5}}{2}\right)
\end{aligned}
$$

where the symmetrization with respect to $\alpha \leftrightarrow \beta, \lambda \leftrightarrow \rho$ and $\mu \leftrightarrow \nu$ is understood. We should now proceed to the explicit calculation. However one quickly realizes that this involves a huge number of terms. To find an orientation among the latter it is very useful to first compute the trace and the divergence of the e.m. tensor in the above formulas. They are connected to the trace and divergence of the full one-loop e.m. tensor by the general formulas of section 1.1.

\subsubsection{The trace}

The trace of (6.33) is

$$
\begin{aligned}
\mathcal{T}_{\mu \alpha \beta \lambda \rho}^{(1 a)^{\mu}}\left(k_{1}, k_{2}\right)= & -\frac{1}{256} \int \frac{d^{4} p}{(2 \pi)^{4}} \int \frac{d^{\delta} \ell}{(2 \pi)^{\delta}} \operatorname{tr}\left(\frac{\not p+\ell}{p^{2}-\ell^{2}}\left(2 p-k_{1}\right)_{\lambda} \gamma_{\rho}\right. \\
& \left.\times \frac{\not p+\ell-\not k_{1}}{\left(p-k_{1}\right)^{2}-\ell^{2}}\left(2 p-2 k_{1}-k_{2}\right)_{\alpha} \gamma_{\beta} \frac{\not p+\ell-\not p}{(p-q)^{2}-\ell^{2}}(2 \not p-\not q) \frac{1+\gamma_{5}}{2}\right) .
\end{aligned}
$$


On the other hand if we first take the trace of (6.31) and then regularize it, we get

$$
\begin{aligned}
\mathcal{T}_{\mu \alpha \beta \lambda \rho}^{(1 b)^{\mu}}\left(k_{1}, k_{2}\right)= & -\frac{1}{256} \int \frac{d^{4} p}{(2 \pi)^{4}} \int \frac{d^{\delta} \ell}{(2 \pi)^{\delta}} \operatorname{tr}\left(\frac{\not p+\ell}{p^{2}-\ell^{2}}\left(2 p-k_{1}\right)_{\lambda} \gamma_{\rho}\right. \\
& \left.\times \frac{\not p+\ell-\not k_{1}}{\left(p-k_{1}\right)^{2}-\ell^{2}}\left(2 p-2 k_{1}-k_{2}\right)_{\alpha} \gamma_{\beta} \frac{\not p+\ell-\not q}{(p-q)^{2}-\ell^{2}}(2 \not p+2 \ell-\not q) \frac{1+\gamma_{5}}{2}\right) .
\end{aligned}
$$

The difference between the two is ${ }^{10}$

$$
\begin{aligned}
\Delta \mathcal{T}_{\mu \alpha \beta \lambda \rho}^{(1)^{\mu}}\left(k_{1}, k_{2}\right)= & -\frac{1}{128} \int \frac{d^{4} p}{(2 \pi)^{4}} \int \frac{d^{\delta} \ell}{(2 \pi)^{\delta}} \operatorname{tr}\left(\frac{\not p+\ell}{p^{2}-\ell^{2}}\left(2 p-k_{1}\right)_{\lambda} \gamma_{\rho}\right. \\
& \left.\times \frac{\not p+\ell-\not \ell_{1}}{\left(p-k_{1}\right)^{2}-\ell^{2}}\left(2 p-2 k_{1}-k_{2}\right)_{\alpha} \gamma_{\beta} \frac{\not p+\ell-\not 1}{(p-q)^{2}-\ell^{2}} \ell \frac{1+\gamma_{5}}{2}\right) .
\end{aligned}
$$

Similar expressions hold for $\mathcal{T}^{(2)}$. Now it is easy to show that (6.34) vanishes along with the analogous expression for $\mathcal{T}^{(2)}$, while (6.35) does not, and in fact the odd-parity part of (6.36) is precisely the anomalous term computed in [22], which, together with the cross term coming form $\mathcal{T}^{(2)}$, gives rise to the Pontryagin anomaly. More precisely, the two terms yield

$$
\mathcal{T}_{\mu \alpha \beta \lambda \rho}^{\mu}\left(k_{1}, k_{2}\right)=\frac{1}{192(4 \pi)^{2}} k_{1}^{\sigma} k_{2}^{\tau}\left(t_{\lambda \rho \alpha \beta \sigma \tau}^{(21)}-t_{\lambda \rho \alpha \beta \sigma \tau}\left(k_{1}^{2}+k_{2}^{2}+k_{1} k_{2}\right)\right)
$$

The tensors $t$ and $t^{(21)}$ were defined in [22]. In [22] the external lines were put on shell (in the de Donder gauge): $k_{1}^{2}=k_{2}^{2}=0$. This is the right thing to do, as we shall see, but it is important to clarify the role of the off-shell terms too. Therefore let us consider nonvanishing external square momenta. While the remaining terms, when inserted into the reconstruction formula (1.4), reproduce the Pontryagin density to order $h^{2}$,

$$
\sim \epsilon^{\mu \nu \lambda \rho}\left(\partial_{\mu} \partial_{\sigma} h_{\nu}^{\tau} \partial_{\lambda} \partial_{\tau} h_{\rho}^{\sigma}-\partial_{\mu} \partial_{\sigma} h_{\nu}^{\tau} \partial_{\lambda} \partial^{\sigma} h_{\tau \rho}\right)+\mathcal{O}\left(h^{3}\right),
$$

the term proportional to $k_{1}^{2}+k_{2}^{2}$ in (6.36) leads to a term proportional to

$$
\epsilon^{\mu \nu \lambda \rho} \partial_{\mu} \square h_{\nu}^{\alpha} \partial_{\lambda} h_{\rho \alpha}
$$

They are both invariant under the Weyl rescaling $\delta h_{\mu \nu}=2 \omega \eta_{\mu \nu}$. Thus the corresponding anomalous terms obtained by integrating (6.37) and (6.38) multiplied by the Weyl parameter $\omega$ are consistent. But while the first gives rise to a true anomaly, the second one must be trivial because there is no covariant cocycle containing the $\epsilon$ tensor beside the Pontryagin one. In fact it is easy to guess the counterterm that cancels it: it is proportional to

$$
\int d^{4} x h \epsilon^{\mu \nu \lambda \rho} \partial_{\mu} \square h_{\nu}^{\alpha} \partial_{\lambda} h_{\rho \alpha}
$$

\footnotetext{
${ }^{10}$ Eqs. (1.4) and (1.6) suggest that the right prescription is (6.35), not (6.34). This has been fully confirmed by the calculations in $2 d$. The anomaly is determined by the $n$-point functions where the entries are one trace of the e.m. tensor and $n-1$ e.m. tensors. We have quoted the 'wrong' formula (6.34) on purpose in order to stress this point.
} 
where $h=h_{\mu}^{\mu}$. But this counterterm breaks invariance under general coordinate transformations, which to lowest order take the form $\delta_{\xi} h_{\mu \nu}=\partial_{\mu} \xi_{\nu}+\partial_{\nu} \xi_{\mu}$ (with $\delta_{\xi} \omega=0$ ). Thus we must expect that off-shell terms break the e.m. tensor conservation. This does not mean that there are true diff anomalies, but simply that we have to subtract counterterms (actually, a lot of them, see below) in order to recover a covariant regularization. In other words taking into account off-shell terms is a very effective way to complicate one's own life, while disregarding them does not spoil the result if our aim is to find a covariant expression of the anomaly. The reason for this is that the equation of motion of gravity in vacuum

$$
\square h_{\mu \nu}-\partial_{\mu} \partial_{\lambda} h_{\nu}^{\lambda}-\partial_{\nu} \partial_{\lambda} h_{\mu}^{\lambda}+\partial_{\mu} \partial_{\nu} h_{\lambda}^{\lambda}=0
$$

is covariant. If we impose the De Donder gauge

$$
2 \partial_{\mu} h_{\lambda}^{\mu}-\partial_{\lambda} h_{\mu}^{\mu}=0
$$

the last three terms in the r.h.s. of (6.40) vanish and the latter reduces to $\square h_{\mu \nu}=0$. Therefore choosing this gauge and putting the external legs on shell (as we have just done) does not break covariance and considerably simplifies the calculations. ${ }^{11}$

\subsubsection{The divergence}

The discussion in the previous subsection raises a problem. For not only can we subtract (6.38) via the counterterm (6.39), but also (6.37) can be subtracted away by means of the counterterm

$$
\sim \int d^{4} x h \epsilon^{\mu \nu \lambda \rho}\left(\partial_{\mu} \partial_{\sigma} h_{\nu}^{\tau} \partial_{\lambda} \partial_{\tau} h_{\rho}^{\sigma}-\partial_{\mu} \partial_{\sigma} h_{\nu}^{\tau} \partial_{\lambda} \partial^{\sigma} h_{\tau \rho}\right)+\mathcal{O}\left(h^{3}\right)
$$

as it is easy to verify. This of course generates new terms in the divergence of the e.m. tensor. Choosing the on-shell option to simplify the problem, they corresponds, in the momentum notation, to the terms

$$
\sim \epsilon_{\beta \rho \sigma \tau} k_{1 \nu} k_{1}^{\sigma} k_{2}^{\tau}\left(k_{1 \lambda} k_{2 \alpha}-\eta_{\alpha \lambda} k_{1} \cdot k_{2}\right)+\{\lambda \leftrightarrow \rho\}+\{\alpha \leftrightarrow \beta\}+\{1 \leftrightarrow 2\}
$$

where the subscript $\nu$, in coordinate representation, is saturated with the diffeomorphism parameter $\xi^{\nu}$.

Let us remark that, when we refer to the lowest order in $h$, any anomaly appears to be trivial and can be subtracted (see what we have done above in $2 d$ ). This is true also for the even parity anomalies, but it is an accident of the approximation. What is decisive about triviality or not of the anomalies is their diff partner. We must arrive at a configuration in which the diff partner of the trace anomaly vanishes. In this case we can conclude that a nonvanishing trace anomaly is nontrivial even if it is expressed at the lowest order in $h$. This expression will be the lowest order expansion of a covariant expression (much as (6.37) is). In conclusion we expect that subtracting away (6.37) by means of (6.42) is a forbidden

\footnotetext{
${ }^{11}$ Sometimes it oversimplifies them, for instance in $2 d$ or in $4 d$ for the 2-point correlator. In such cases there is no way but doing the calculations in full, as we have done above.
} 
operation (it breaks covariance). But it is important to verify it by a direct calculation. This is what we intend to do in the sequel.

The relevant lowest order contribution to $\left\langle\left\langle\nabla^{\mu} T_{\mu \nu}\right\rangle\right\rangle$, see (1.8), comes from the 3-point function $\left\langle 0\left|\mathcal{T}\left\{\partial^{\mu} T_{\mu \nu}(x) T_{\lambda \rho}(y) T_{\alpha \beta}(z)\right\}\right| 0\right\rangle$. The latter corresponds to two graphs, the bubble and the triangle ones (see [22]). The bubble graph contribution vanishes. The triangle contribution is given by

$$
\begin{aligned}
& q^{\mu} \mathcal{T}_{\mu \nu \lambda \rho \alpha \beta}^{(1)}\left(k_{1}, k_{2}\right)=-\frac{1}{512} \int \frac{d^{4} p}{(2 \pi)^{4}} \operatorname{tr}\left[\left(\frac{1}{\not p}\left(\left(2 p-k_{1}\right)_{\lambda} \gamma_{\rho}+(\lambda \leftrightarrow \rho)\right) \frac{1}{\not p-\not k_{1}}\right.\right. \\
& \left.\left.\times\left(\left(2 p-2 k_{1}-k_{2}\right)_{\alpha} \gamma_{\beta}+(\alpha \leftrightarrow \beta)\right) \frac{1}{\not p-\not q}\left((2 p-q) \cdot q \gamma_{\nu}+(2 p-q)_{\nu} q\right)\right) \frac{1+\gamma_{5}}{2}\right]
\end{aligned}
$$

to which the cross contribution $q^{\mu} \mathcal{T}_{\mu \nu \alpha \beta \lambda \rho}^{(1)}\left(k_{2}, k_{1}\right)$ has to be added. We regulate the integral as usual with an extra dimensional momentum $\ell$ and introduce Feynman parameters as needed. After a rather lengthy algebra, in particular with explicit use of the identity

$$
\eta_{\mu \nu} \epsilon_{\lambda \rho \sigma \tau}-\eta_{\mu \lambda} \epsilon_{\nu \rho \sigma \tau}+\eta_{\mu \rho} \epsilon_{\nu \lambda \sigma \tau}-\eta_{\mu \sigma} \epsilon_{\nu \lambda \rho \tau}+\eta_{\mu \tau} \epsilon_{\nu \lambda \rho \sigma}=0
$$

the regularized (6.44) can be recast into the form

$$
\begin{aligned}
& \mathcal{D}_{\nu \lambda \rho \alpha \beta}^{(1)}\left(k_{1}, k_{2}\right) \equiv q^{\mu}\left(\mathcal{T}_{\mu \nu \lambda \rho \alpha \beta}^{(1)}\left(k_{1}, k_{2}\right)+\mathcal{T}_{\mu \nu \alpha \beta \lambda \rho}^{(1)}\left(k_{2}, k_{1}\right)\right) \\
& =\frac{i}{256} \int_{0}^{1} d x \int_{0}^{1-x} d y \int \frac{d^{4} p}{(2 \pi)^{4}} \int \frac{d^{\delta} \ell}{(2 \pi)^{\delta}}\left[-\epsilon_{\nu \beta \sigma \tau}\left(p_{\rho} k_{1}^{\sigma} k_{2}^{\tau}+\left(k_{1 \rho} k_{2}^{\tau}+k_{2 \rho} k_{1}^{\tau}+2 k_{2}^{\tau} p_{\rho}\right) p^{\sigma}\right)\right. \\
& \quad+\epsilon_{\nu \beta \rho \tau}\left(p^{2}\left(k_{1}+k_{2}-p\right)^{\tau}+p \cdot k_{1} k_{2}^{\tau}-p \cdot k_{2} k_{1}^{\tau}+\left(k_{2}-2 p\right) \cdot k_{1} p^{\tau}\right)+\epsilon_{\nu \sigma \tau \kappa} \eta_{\beta \rho} p^{\sigma} k_{1}^{\tau} k_{2}^{\kappa} \\
& \left.\quad+\epsilon_{\nu \rho \sigma \tau}\left(p_{\beta} k_{1}^{\tau} k_{2}^{\sigma}+p^{\sigma}\left(k_{1 \beta} k_{2}^{\tau}+k_{2 \beta} k_{1}^{\tau}-2 p_{\beta} k_{1}^{\tau}\right)\right)+\ell^{2} \epsilon_{\nu \beta \rho \tau}\left(p+k_{1}-k_{2}\right)^{\tau}\right] \\
& \quad \times \frac{2 p \cdot\left(k_{1}+k_{2}\right)\left(2 p+k_{1}\right)_{\lambda}\left(2 p-k_{2}\right)_{\alpha}}{\left[\left(p+x k_{1}-y k_{2}\right)^{2}+2 x y k_{1} \cdot k_{2}-\ell^{2}\right]^{3}} .
\end{aligned}
$$

This expression does not contain any of the terms (6.43), but of course this is not enough. We have to prove that all the terms in (6.46) either vanish or are trivial in the sense that they can be canceled by counterterms that are Weyl invariant. This analysis is carried out in appendix E, where counterterms are constructed which cancel all the nonvanishing terms in (6.46) without altering the result of the trace anomaly calculation. Thus the lowest order expression (6.37) cannot be canceled (except at the price of breaking diffeomorphism invariance) and is a genuine covariant expression. It represents the lowest order approximation of the Pontryagin density.

\subsection{3 (Partial) conclusion}

The results obtained in this section fully confirm those of $[19,20,22]$. The apparent contradiction inherent in the fact that the "bare" parity-odd correlator of three energymomentum tensors vanishes will be explained in the next section. Here we would like to draw some conclusion on the regularized e.m. tensor 3-point function. We have seen that the trace and the traceless part of the correlator must be regularized separately. The 
traceless part of the correlator can be regularized starting from (6.33). We would like to be able to conclude that the regularized traceless part coincides with the "bare" part, i.e. it vanishes, but in order to justify this conclusion the calculations are very challenging, because it is not enough to regularize and compute (6.33), but we must also take into account all the counterterms (with the exact coefficients) that we have subtracted in order to guarantee covariance, see appendix E. This can realistically be done only with a computer algebra program. For the time being, although we believe the regularized traceless part of the correlator vanishes, we leave its proof as an open problem.

Finally a comment on the parity-even part of the 3-point e.m. tensor correlator. The calculation of the trace and divergence involves many more terms than in the parity-odd part, but it does not differ in any essential way from it. Also in the parity-even part it is necessary to introduce counterterms in order to guarantee covariance and the correct final expression for the trace anomaly. On the other hand this is pretty clear already in the $2 d$ case, as we have shown above. Since the results for the parity-even part of the 3 -point function, both "bare" and regularized, [3, 4], and relevant even-parity anomalies are well-known, see [29], we dispense with an explicit calculation.

\section{The ugly duckling anomaly}

The title is due to the non-overwhelming consideration met so far by the Pontryagin trace anomaly. Needless to say its presence in the free chiral fermion model is at first sight surprising. The basic ingredient to evaluate this anomaly in the Feynman diagram approach is traditionally the triangle diagram, which can be seen as the lowest order approximation of the 3-point correlator, whose entries are one e.m. trace and two e.m. tensors. On the other hand, since the "bare" parity-odd part of the 3-point correlator of the e.m. tensor vanishes on the basis of very general considerations of symmetry, it would seem that even the triangle diagram contributions should vanish, because the regularization of zero should be zero.

The remark made in connection with formulas (6.34), (6.35) and (6.36) may seem to add strength to this argument because it leaves the impression that the Pontryagin anomaly is something we can do without. After all its existence in the 3-point correlators is related to the order in which we regularize. One might argue that if we regularize in a specific order the anomaly disappears, but this is not the case. First of all we remark that what one does in all kind of anomalies is to regularize the divergence of a current or of the e.m. tensor, or the trace of the latter, rather than regularizing the current or the e.m. tensor and then taking the divergence or the trace thereof. In other words the regularization should be done independently for each irreducible component that enters into play. But, even forgetting this, in order to make a decision about such an ambiguous occurrence one must resort to some consistency argument, and this is what we will do below.

In fact the apparent contradiction is based on a misunderstanding, which consists in assuming that the (unregulated) 3-point correlator in the coordinate representation is the sole ingredient of the anomaly. This is not true. ${ }^{12}$ The 3 -point correlator of the energy-

\footnotetext{
${ }^{12}$ We remark that the parity-even 3-point correlator of the e.m. trace and two e.m. tensors also vanishes for non-coincident points, but this does not prevent the even parity anomaly from being nonvanishing.
} 
momentum tensor is one of the possible markers of the trace anomaly, but, as we shall see, there are infinite many of them and consistency demands that they all agree (the more so if the correlator is unregulated). Let us start with by clarifying this point.

In subsection 1.1 we have shown how to reconstruct the full one-loop e.m. tensor starting from the one-loop correlators of the e.m. tensors, see (1.4). What matters here is that the full one-loop e.m. tensor contains the information about the e.m. tensor correlators with any number of entries. The first non-trivial one corresponds of course to $n=2$.

Now let us apply the reconstruction formula (1.4) to a single chiral fermion theory. Classically the energy-momentum tensor for a left-handed fermion is

$$
T_{\mu \nu}^{(L)}=\frac{i}{4} \overline{\psi_{L}} \gamma_{\mu} \stackrel{\leftrightarrow}{\partial}_{\nu} \psi_{L}+\{\mu \leftrightarrow \nu\}
$$

which is both conserved and traceless on shell. An analogous expression holds for a righthanded fermion. It has been proved in general (and we have shown it above) that the (unregulated) parity-odd 3-point function in the coordinate representation vanishes. Thus let us ask ourselves what would happen if parity-odd amplitudes

$$
\left\langle 0\left|\mathcal{T}\left\{T_{\mu_{1} \nu_{1}}^{(L)}\left(x_{1}\right) \ldots T_{\mu_{n} \nu_{n}}^{(L)}\left(x_{n}\right)\right\}\right| 0\right\rangle_{\text {odd }}
$$

to all orders were to vanish. We would have the same also for the right handed counterpart, while the even-parity amplitudes are equal. Therefore the difference

$$
\left\langle\left\langle T_{\mu \nu}^{(L)}(x)\right\rangle\right\rangle-\left\langle\left\langle T_{\mu \nu}^{(R)}(x)\right\rangle\right\rangle=0
$$

This would imply that the quantum analog of $\bar{\psi} \gamma_{\mu} \gamma_{5} \stackrel{\leftrightarrow}{\partial}_{\nu} \psi+\{\mu \leftrightarrow \nu\}$ would vanish identically. This is nonsense, and means that the vanishing of the parity-odd 3-point function is an accidental occurrence and that the ("bare") parity-odd amplitudes will generically be non-vanishing. ${ }^{13}$ Inserting now these results in the reconstruction formula (1.4) and resumming the series we would reconstruct the parity-odd anomaly. Let us apply this to the trace of the quantum energy-momentum tensor. Since the parity-odd amplitudes are generically nonvanishing we would obtain a nonvanishing trace anomaly. Now the only possible covariant parity-odd anomaly is the Pontryagin density

$$
P=\frac{1}{2}\left(\epsilon^{n m l k} \mathcal{R}_{n m p q} \mathcal{R}_{l k}^{p q}\right)
$$

whose first nonvanishing contribution is quadratic in $h_{\mu \nu}$

$$
\epsilon^{\mu \nu \lambda \rho} R_{\mu \nu}^{\sigma \tau} R_{\lambda \rho \sigma \tau}=2 \epsilon^{\mu \nu \lambda \rho}\left(\partial_{\mu} \partial_{\sigma} h_{\nu}^{\tau} \partial_{\lambda} \partial_{\tau} h_{\rho}^{\sigma}-\partial_{\mu} \partial_{\sigma} h_{\nu}^{\tau} \partial_{\lambda} \partial^{\sigma} h_{\tau \rho}\right)+\ldots
$$

and can come only from the parity-odd 3-point correlator. But, if the latter vanishes, we would get an incomplete, and therefore non-covariant, expression for this anomaly.

The conclusion of this argument is: covariance (and consistency) requires that, even if the (unregulated) parity-odd 3-point function in the coordinate representation vanishes,

\footnotetext{
${ }^{13}$ The analogue of the parity-odd 3-point correlator vanishing theorem does not exist for generic amplitudes.
} 
the corresponding regularized counterpart must be non-vanishing. This is precisely what was found in [22] with (regularized) Feynman diagram techniques.

The existence of the Pontryagin anomaly is confirmed also by other methods of calculation: the heat kernel method, see $[20,22]$ and references therein, and the mass regularization of [30], although the latter method have not been applied with the same accuracy as the dimensional regularization in the present paper. We should mention also the dispersive method which uses unitarity as an input. Of course we do not expect this method to reproduce this anomaly, which violates unitarity, [22]. In fact using such a method would be a reversal of the burden of proof. The dispersive argument is very elegant and powerful, [12, 25, 31], but it assume unitarity. Unitarity is normally given for granted and assumed by default. But the case presented in this paper is precisely an example in which this cannot be done.

Finally we would like to notice that the so-called Delbourgo-Salam anomaly, [30], i.e. the anomaly in the divergence of the chiral current $j_{\mu 5}=i \bar{\psi} \gamma_{\mu} \gamma_{5} \psi$, is determined by a term $(6.34)$ in which the factor $(2 \not p-q)$ is replaced by $q$. If, in such a term, we rewrite $q$ as $2 \not p-(2 \not p-\not q)$, we see that the second part reproduces the Pontryagin anomaly we have computed, while the term containing $2 \not p$, once regularized, is easily seen to vanish. In other words the Pontryagin trace anomaly and the Delbourgo-Salam chiral anomaly come from the same term.

\section{Conclusions}

In conclusion, let us summarize what was reviewed and what was shown in this paper. Our paradigm is always the theory of a free chiral fermion, thus every time that we refer to Feynman diagram techniques or Wick theorem, we are making reference to these techniques applied to this specific model.

We started in sections 2, 3 and 4 by reviewing the regularization of the 2-point function of e.m. tensors in $2 d$, using both differential regularization and dimensional regularization of the expression obtained with Feynman diagrams. Demanding the correlator to satisfy the Ward identity for diffeomorphism invariance we obtain a violation of the Ward identity for conformal invariance and we recover the known result of the $2 d$ trace anomaly. In section 5 the analogous result was shown also in $4 d$ where the situation is different because we are able to regularize the correlator in such a way that both Ward identities are satisfied.

In section 6, moving to the 3-point function of e.m. tensors in $4 d$, we first noted a discrepancy between the computations in momentum space through Feynman diagrams and the computation in coordinate space using the Wick theorem. The direct c omputation through Wick theorem tells us that there is no (unregulated) parity-odd contribution in the 3-point correlator of e.m. tensors for the free chiral fermion. This result is indeed in agreement with the general fact that in $4 d$ there are no parity-odd contribution in the correlation function of three e.m. tensors which was reviewed in section 6.1. With this fact in hand one could try to regularize this correlator with the techniques of differential regularization and would be obliged to conclude that there is no parity-odd trace anomaly simply because there is no parity-odd contribution to be regularized. On the other hand, 
by doing the computation in momentum space with Feynman diagram techniques we do find a parity-odd trace anomaly. Is this result forced to be wrong?

We argued in section 7 that these results can perfectly coexist and the result in coordinate space by no means is a no-go for the existence of the Pontryagin anomaly.

\section{Acknowledgments}

BLS would like to thank Alexander Zhiboedov for useful discussion. LB would like to thank Roberto Auzzi, Carl Bender, Maro Cvitan, Holger Nielsen, Silvio Pallua, Predrag Dominis-Prester, Ivica Smolić, Alexander Sorin and in particular Adam Schwimmer for very interesting discussions. ADP acknowledges CNPq and CAPES for financial support and, in particular, CAPES for supporting his stay at SISSA and the Theoretical Particle Physics division of SISSA for supporting and hospitality. This work has been supported in part by the Croatian Science Foundation under the project 8946.

\section{A Direct computation for a chiral fermion in $2 d$}

Consider a free chiral fermion $\psi_{L}$ in $2 d$ which has the 2-point function

$$
\left\langle\psi_{L}(x) \overline{\psi_{L}}(y)\right\rangle=\frac{i}{2 \pi} \frac{\gamma \cdot(x-y)}{(x-y)^{2}} P_{L}, \quad P_{L}=\frac{1-\gamma_{*}}{2},
$$

and the e.m. tensor

$$
T_{\mu \nu}=\frac{i}{4}\left(\overline{\psi_{L}} \gamma_{\mu} \stackrel{\leftrightarrow}{\partial}_{\nu} \psi_{L}+\mu \leftrightarrow \nu\right)
$$

Before proceeding with the calculation let us recall some definitions:

$$
\left\{\gamma^{\mu}, \gamma^{\nu}\right\}=2 \eta^{\mu \nu} \Rightarrow\left(\gamma^{0}\right)^{2}=1, \quad\left(\gamma^{i}\right)^{2}=-1
$$

Clearly, $\gamma^{0}=\gamma_{0}$ and $\gamma^{i}=-\gamma_{i}$. For an arbitrary dimension $D$ the analogous of $\gamma_{5}$ will be denoted $\gamma_{*}$ and it is given by $\gamma_{*}=(-i)^{\frac{D}{2}+1} \gamma_{0} \gamma_{1} \ldots \gamma_{D-1}$, which for $D=2$ means $\gamma_{*}=-\gamma_{0} \gamma_{1}$.

It is straightforward to check that the following relations are true:

$$
\gamma_{\mu}=\epsilon_{\mu \nu} \gamma^{\nu} \gamma_{*}, \quad \epsilon_{\mu \nu} \gamma^{\nu}=\gamma_{\mu} \gamma_{*},
$$

where we are using the convention where $\epsilon_{01}=1$. It follows

$$
\operatorname{tr}\left(\gamma_{\mu} \gamma_{\nu} \gamma_{*}\right)=-2 \epsilon_{\mu \nu}
$$

Our purpose is to compute the 2-point of the em tensor in the theory (A.1). Since we are dealing with a simple free theory we can use the Wick theorem.

The non-zero part of the correlation function comes from the

$$
\left\langle T_{\mu \nu}(x) T_{\rho \sigma}(y)\right\rangle=\frac{1}{16}\left\langle: \bar{\psi}_{L} \gamma_{\mu} \stackrel{\leftrightarrow}{\partial}_{\nu} \psi_{L}:(x): \bar{\psi}_{L} \gamma_{\rho} \stackrel{\leftrightarrow}{\partial}_{\sigma} \psi_{L}:(y)\right\rangle+\text { sym. }
$$


which is given by the full contraction of this object, namely

$$
\begin{aligned}
\left\langle T_{\mu \nu}(x) T_{\rho \sigma}(y)\right\rangle= & \frac{1}{16(2 \pi)^{2}}\left(\operatorname{tr}\left[\gamma_{\mu} \partial_{\nu}^{x}\left\langle\psi_{L}(x) \bar{\psi}_{L}(y)\right\rangle \gamma_{\rho} \partial_{\sigma}^{y}\left\langle\psi_{L}(y) \bar{\psi}_{L}(x)\right\rangle\right]+\cdots\right) \\
& + \text { sym. }
\end{aligned}
$$

where the ellipsis stand for the three other ways of organizing the derivatives. We may use the translational invariance of this correlator to shift $x \rightarrow x-y$ and $y \rightarrow 0$. For simplicity we will relabel $x-y$ calling it simply $x$. Since the correlation function is simply a function of $x-y, \partial^{y}=-\partial^{x}$. Let us also remark that $\left\langle\psi_{L}(x) \bar{\psi}_{L}(y)\right\rangle=-\left\langle\psi_{L}(y) \bar{\psi}_{L}(x)\right\rangle$. Thus, we can exchange all the derivatives on $y$ by derivatives on $x$ and the correlations functions $\left\langle\psi_{L}(y) \bar{\psi}_{L}(x)\right\rangle$ by $\left\langle\psi_{L}(x) \bar{\psi}_{L}(y)\right\rangle$, which, due to translational invariance, can be written as $\left\langle\psi_{L}(x-y) \bar{\psi}_{L}(0)\right\rangle$. Therefore,

$$
\begin{aligned}
\left\langle T_{\mu \nu}(x) T_{\rho \sigma}(y)\right\rangle= & \frac{1}{16(2 \pi)^{2}}\left(\operatorname{tr}\left[\gamma_{\mu} \partial_{\nu}\left\langle\psi_{L}(x) \bar{\psi}_{L}(0)\right\rangle \gamma_{\rho} \partial_{\sigma}\left\langle\psi_{L}(x) \bar{\psi}_{L}(0)\right\rangle\right]+\cdots\right) \\
& + \text { sym. }
\end{aligned}
$$

Using the expression for the 2-point function (A.1) we have

$\operatorname{tr}\left[\gamma_{\mu} \partial_{\nu}\left\langle\psi_{L}(x) \bar{\psi}_{L}(0)\right\rangle \gamma_{\rho} \partial_{\sigma}\left\langle\psi_{L}(x) \bar{\psi}_{L}(0)\right\rangle\right]=\frac{1}{(2 \pi)^{2}} \partial_{\nu}\left(\frac{x^{\alpha}}{x^{2}}\right) \partial_{\sigma}\left(\frac{x^{\beta}}{x^{2}}\right) \operatorname{tr}\left(\gamma_{\mu} \gamma_{\alpha} \gamma_{\rho} \gamma_{\beta} P_{L}\right)$,

and analogously for the other terms. One should notice that

$$
\operatorname{tr}\left(\gamma_{\mu} \gamma_{\beta} \gamma_{\rho} \gamma_{\alpha} P_{L}\right)=\operatorname{tr}\left(\gamma_{\rho} \gamma_{\alpha} \gamma_{\mu} \gamma_{\beta} P_{L}\right)
$$

and we are able to rewrite our correlation function as

$$
\begin{aligned}
\left\langle T_{\mu \nu}(x) T_{\rho \sigma}(y)\right\rangle= & \frac{1}{16} \frac{1}{(2 \pi)^{2}}\left[\partial_{\nu}\left(\frac{x^{\alpha}}{x^{2}}\right) \partial_{\sigma}\left(\frac{x^{\beta}}{x^{2}}\right)-\left(\frac{x^{\alpha}}{x^{2}}\right) \partial_{\nu} \partial_{\sigma}\left(\frac{x^{\beta}}{x^{2}}\right)\right] \\
& \times\left[\operatorname{tr}\left(\gamma_{\mu} \gamma_{\alpha} \gamma_{\rho} \gamma_{\beta} P_{L}\right)+\mu \leftrightarrow \rho\right]+\operatorname{sym} .
\end{aligned}
$$

Exchanging the position of $\gamma_{\alpha}$ and $\gamma_{\rho}$ in $\operatorname{tr}\left(\gamma_{\mu} \gamma_{\alpha} \gamma_{\rho} \gamma_{\beta} P_{L}\right)$ we have

$$
\operatorname{tr}\left(\gamma_{\mu} \gamma_{\alpha} \gamma_{\rho} \gamma_{\beta} P_{L}\right)=2 \eta_{\alpha \rho} \operatorname{tr}\left(\gamma_{\mu} \gamma_{\beta} P_{L}\right)-\operatorname{tr}\left(\gamma_{\mu} \gamma_{\rho} \gamma_{\alpha} \gamma_{\beta} P_{L}\right)
$$

Thus

$$
\begin{aligned}
\operatorname{tr}\left(\gamma_{\mu} \gamma_{\alpha} \gamma_{\rho} \gamma_{\beta} P_{L}\right)+\mu \leftrightarrow \rho & =2 \eta_{\alpha \rho} \operatorname{tr}\left(\gamma_{\mu} \gamma_{\beta} P_{L}\right)+2 \eta_{\alpha \mu} \operatorname{tr}\left(\gamma_{\rho} \gamma_{\beta} P_{L}\right)-\operatorname{tr}\left(\left\{\gamma_{\mu}, \gamma_{\rho}\right\} \gamma_{\alpha} \gamma_{\beta} P_{L}\right) \\
& =2\left[\eta_{\alpha \rho} \operatorname{tr}\left(\gamma_{\mu} \gamma_{\beta} P_{L}\right)+\eta_{\alpha \mu} \operatorname{tr}\left(\gamma_{\rho} \gamma_{\beta} P_{L}\right)-\eta_{\mu \rho} \operatorname{tr}\left(\gamma_{\alpha} \gamma_{\beta} P_{L}\right)\right]
\end{aligned}
$$

The trace of $\gamma_{\mu} \gamma_{\nu} P_{L}$ is straightforward to compute (see appendix):

$$
\operatorname{tr}\left(\gamma_{\mu} \gamma_{\nu} P_{L}\right)=\frac{1}{2}\left[\operatorname{tr}\left(\gamma_{\mu} \gamma_{\nu}\right)-\operatorname{tr}\left(\gamma_{\mu} \gamma_{\nu} \gamma_{*}\right)\right]=\eta_{\mu \nu}+\epsilon_{\mu \nu}
$$

Therefore

$$
\operatorname{tr}\left(\gamma_{\mu} \gamma_{\alpha} \gamma_{\rho} \gamma_{\beta} P_{L}\right)+\mu \leftrightarrow \rho=2\left(\eta_{\alpha \rho} \eta_{\mu \beta}+\eta_{\alpha \mu} \eta_{\rho \beta}-\eta_{\mu \rho} \eta_{\alpha \beta}\right)+2\left(\eta_{\alpha \rho} \epsilon_{\mu \beta}+\eta_{\alpha \mu} \epsilon_{\rho \beta}-\eta_{\mu \rho} \epsilon_{\alpha \beta}\right) .
$$


It turns out that we are able to rewrite $\eta_{\mu \rho} \epsilon_{\alpha \beta}$ as

$$
\eta_{\mu \rho} \epsilon_{\alpha \beta}=\frac{1}{2}\left(\eta_{\alpha \mu} \epsilon_{\rho \beta}-\eta_{\beta \mu} \epsilon_{\rho \alpha}+\eta_{\alpha \rho} \epsilon_{\mu \beta}-\eta_{\beta \rho} \epsilon_{\mu \alpha}\right)
$$

and using this expression we may rewrite (A.9) as

$$
\begin{aligned}
\operatorname{tr}\left(\gamma_{\mu} \gamma_{\alpha} \gamma_{\rho} \gamma_{\beta} P_{L}\right)+\mu \leftrightarrow \rho= & 2\left(\eta_{\alpha \rho} \eta_{\mu \beta}+\eta_{\alpha \mu} \eta_{\rho \beta}-\eta_{\mu \rho} \eta_{\alpha \beta}\right) \\
& +\left(\eta_{\alpha \rho} \epsilon_{\mu \beta}+\eta_{\alpha \mu} \epsilon_{\rho \beta}+\eta_{\beta \mu} \epsilon_{\rho \alpha}+\eta_{\beta \rho} \epsilon_{\mu \alpha}\right) .
\end{aligned}
$$

Using (A.10) we can compute (A.8) and we find the parity-odd part

$$
\begin{aligned}
& \left\langle T_{\mu \nu}(x) T_{\rho \sigma}(0)\right\rangle_{\text {odd }}= \\
& -\frac{1}{4 \pi^{2}}\left(\frac{\epsilon_{\mu \alpha} x^{\alpha} x_{\nu} x_{\rho} x_{\sigma}}{x^{8}}+\frac{\epsilon_{\nu \alpha} x^{\alpha} x_{\mu} x_{\rho} x_{\sigma}}{x^{8}}+\frac{\epsilon_{\rho \alpha} x^{\alpha} x_{\mu} x_{\nu} x_{\sigma}}{x^{8}}+\frac{\epsilon_{\sigma \alpha} x^{\alpha} x_{\mu} x_{\nu} x_{\rho}}{x^{8}}\right. \\
& -\frac{\epsilon_{\mu \alpha} \eta_{\rho \nu} x^{\alpha} x_{\sigma}}{4 x^{6}}-\frac{\epsilon_{\mu \alpha} \eta_{\sigma \nu} x^{\alpha} x_{\rho}}{4 x^{6}}-\frac{\epsilon_{\nu \alpha} \eta_{\rho \mu} x^{\alpha} x_{\sigma}}{4 x^{6}}-\frac{\epsilon_{\nu \alpha} \eta_{\sigma \mu} x^{\alpha} x_{\rho}}{4 x^{6}} \\
& \left.-\frac{\epsilon_{\rho \alpha} \eta_{\mu \sigma} x^{\alpha} x_{\nu}}{4 x^{6}}-\frac{\epsilon_{\rho \alpha} \eta_{\nu \sigma} x^{\alpha} x_{\mu}}{4 x^{6}}-\frac{\epsilon_{\sigma \alpha} \eta_{\mu \rho} x^{\alpha} x_{\nu}}{4 x^{6}}-\frac{\epsilon_{\sigma \alpha} \eta_{\nu \rho} x^{\alpha} x_{\mu}}{4 x^{6}}\right) .
\end{aligned}
$$

As a matter of fact, out of this computation we find that the parity-even part matches (2.1) with $c=1 / 4 \pi^{2}$, in agreement with $[3,4]$. The expression (A.11) is traceless, conserved and can be written as

$$
\left\langle T_{\mu \nu}(x) T_{\rho \sigma}(0)\right\rangle_{\text {odd }}=\frac{1}{32 \pi^{2}}\left(\epsilon_{\alpha \mu} T_{\nu \rho \sigma}^{\alpha}+\epsilon_{\alpha \nu} T_{\mu \rho \sigma}^{\alpha}+\epsilon_{\alpha \rho} T_{\mu \nu \sigma}^{\alpha}+\epsilon_{\alpha \rho} T_{\mu \nu \rho}{ }^{\alpha}\right),
$$

where $T_{\mu \nu \rho \sigma}$ is given by the expression (3.1). Hence (A.11) agrees with the null cone result.

\section{B The chiral fermion model in $4 d$}

In this appendix we summarize the formalism and notation of [22], concerning the free chiral fermion model minimally coupled to gravity. The action is

$$
S=\int d^{4} x \sqrt{|g|}\left[\frac{i}{2} \overline{\psi_{R}} \gamma^{\mu} \stackrel{\leftrightarrow}{\partial}_{\mu} \psi_{R}-\frac{1}{4} \epsilon^{\mu a b c} \omega_{\mu a b} \overline{\psi_{R}} \gamma_{c} \gamma_{5} \psi_{R}\right]
$$

where it is understood that the derivative applies to $\psi_{L}$ and $\overline{\psi_{L}}$ only. We have used the relation $\left\{\gamma^{a}, \Sigma^{b c}\right\}=i \epsilon^{a b c d} \gamma_{d} \gamma_{5}$. Now one expands

$$
e_{\mu}^{a}=\delta_{\mu}^{a}+\chi_{\mu}^{a}+\ldots, \quad e_{a}^{\mu}=\delta_{a}^{\mu}+\hat{\chi}_{a}^{\mu}+\ldots, \quad \text { and } \quad g_{\mu \nu}=\eta_{\mu \nu}+h_{\mu \nu}+\ldots .
$$

Using the defining relations of metric and vierbein one finds

$$
\hat{\chi}_{\nu}^{\mu}=-\chi_{\nu}^{\mu} \quad \text { and } \quad h_{\mu \nu}=2 \chi_{\mu \nu} .
$$

The spin connection $\omega_{m}=\omega_{m}^{a b} \Sigma_{a b}$, where $\Sigma_{a b}=\frac{1}{4}\left[\gamma_{a}, \gamma_{b}\right]$ are the Lorentz generators, to lowest order is

$$
\omega_{\mu a b} \epsilon^{\mu a b c}=-\epsilon^{\mu a b c} \partial_{\mu} \chi_{a \lambda} \chi_{b}^{\lambda}+\ldots
$$


Therefore up to second order the action can be written

$$
S=\int d^{4} x\left[\frac{i}{2}\left(\delta_{a}^{\mu}-\chi_{a}^{\mu}\right) \overline{\psi_{L}} \gamma^{a} \stackrel{\leftrightarrow}{\partial}_{\mu} \psi_{L}+\frac{1}{4} \epsilon^{\mu a b c} \partial_{\mu} \chi_{a \lambda} \chi_{b}^{\lambda} \bar{\psi}_{L} \gamma_{c} \gamma_{5} \psi_{L}\right]
$$

Splitting it into free and interacting parts, one can extract Feynman rules. The fermion propagator is

$$
\frac{i}{p+i \epsilon}
$$

The two-fermion-one-graviton vertex $\left(V_{f f g}\right)$

$$
-\frac{i}{8}\left[\left(p-p^{\prime}\right)_{\mu} \gamma_{\nu}+\left(p-p^{\prime}\right)_{\nu} \gamma_{\mu}\right] \frac{1+\gamma_{5}}{2}
$$

The two-fermion-two-graviton vertex $\left(V_{f f g g}\right)$ is

$$
\frac{1}{64} t_{\mu \nu \mu^{\prime} \nu^{\prime} \kappa \lambda}\left(k-k^{\prime}\right)^{\lambda} \gamma^{\kappa} \frac{1+\gamma_{5}}{2}
$$

where

$$
t_{\mu \nu \mu^{\prime} \nu^{\prime} \kappa \lambda}=\eta_{\mu \mu^{\prime}} \epsilon_{\nu \nu^{\prime} \kappa \lambda}+\eta_{\nu \nu^{\prime}} \epsilon_{\mu \mu^{\prime} \kappa \lambda}+\eta_{\mu \nu^{\prime}} \epsilon_{\nu \mu^{\prime} \kappa \lambda}+\eta_{\nu \mu^{\prime}} \epsilon_{\mu \nu^{\prime} \kappa \lambda}
$$

\section{Regularization formulas in $2 d$ and $4 d$}

In this appendix we collect the regularized integrals that are needed to evaluate the Feynman diagrams in the text both in $2 d$ and $4 d$. The integrals below are Euclidean integrals. They are an intermediate results needed in order to compute the Feynman diagrams in the text. Since the starting points and the final results are Lorentzian, it is understood that one has to do the appropriate Wick rotations in order to be able to use them.

In $2 d$, after introducing $\delta$ extra dimensions in the internal momentum and a Feynman parameter $u(0 \leq u \leq 1)$, in the limit $\delta \rightarrow 0$, we have

$$
\begin{aligned}
& \int \frac{d^{2} p}{(2 \pi)^{2}} \int \frac{d^{\delta} \ell}{(2 \pi)^{\delta}} \frac{\ell^{2}}{\left(p^{2}+\ell^{2}+\Delta\right)^{2}}=-\frac{1}{4 \pi} \\
& \int \frac{d^{2} p}{(2 \pi)^{2}} \int \frac{d^{\delta} \ell}{(2 \pi)^{\delta}} \frac{\ell^{2} p^{2}}{\left(p^{2}+\ell^{2}+\Delta\right)^{2}}=\frac{1}{4 \pi} \Delta
\end{aligned}
$$

and

$$
\begin{aligned}
& \int \frac{d^{2} p}{(2 \pi)^{2}} \int \frac{d^{\delta} \ell}{(2 \pi)^{\delta}} \frac{p^{2}}{\left(p^{2}+\ell^{2}+\Delta\right)^{2}}=\frac{1}{4 \pi} \frac{1}{\Delta} \\
& \int \frac{d^{2} p}{(2 \pi)^{2}} \int \frac{d^{\delta} \ell}{(2 \pi)^{\delta}} \frac{p^{2}}{\left(p^{2}+\ell^{2}+\Delta\right)^{2}}=\frac{1}{4 \pi}\left(-\frac{2}{\delta}-\gamma+\log (4 \pi)-\log \Delta\right) \\
& \int \frac{d^{2} p}{(2 \pi)^{2}} \int \frac{d^{\delta} \ell}{(2 \pi)^{\delta}} \frac{p^{4}}{\left(p^{2}+\ell^{2}+\Delta\right)^{2}}=\frac{1}{2 \pi} \Delta\left(\frac{2}{\delta}-1+\gamma-\log (4 \pi)+\log \Delta\right)
\end{aligned}
$$

where $\Delta=u(1-u) k^{2}$. 
Proceeding in the same way in $4 d$, with two Feynman parameters $u$ and $v$, in the limit $\delta \rightarrow 0$, beside (6.25), we find

$$
\begin{aligned}
& \int \frac{d^{4} p}{(2 \pi)^{4}} \int \frac{d^{\delta} \ell}{(2 \pi)^{\delta}} \frac{p^{2}}{\left(p^{2}+\ell^{2}+\Delta\right)^{3}}=\frac{1}{(4 \pi)^{2}}\left(-\frac{2}{\delta}-\gamma+\log (4 \pi)-\log \Delta\right) \\
& \int \frac{d^{4} p}{(2 \pi)^{4}} \int \frac{d^{\delta} \ell}{(2 \pi)^{\delta}} \frac{p^{4}}{\left(p^{2}+\ell^{2}+\Delta\right)^{3}}=\frac{\Delta}{2(4 \pi)^{2}}\left(-\frac{2}{\delta}-\gamma+4+\log (4 \pi)-\log \Delta\right)
\end{aligned}
$$

and

$$
\begin{aligned}
& \int \frac{d^{4} p}{(2 \pi)^{4}} \int \frac{d^{\delta} \ell}{(2 \pi)^{\delta}} \frac{\ell^{2}}{\left(p^{2}+\ell^{2}+\Delta\right)^{3}}=-\frac{1}{2(4 \pi)^{2}} \\
& \int \frac{d^{4} p}{(2 \pi)^{4}} \int \frac{d^{\delta} \ell}{(2 \pi)^{\delta}} \frac{\ell^{2} p^{2}}{\left(p^{2}+\ell^{2}+\Delta\right)^{3}}=\frac{1}{(4 \pi)^{2}} \Delta
\end{aligned}
$$

where $\Delta=u(1-u) k_{1}+v(1-v) k_{2}+2 u v k_{1} k_{2}$.

\section{Fourier transforms}

In this appendix we expand on the results of section (6.3). Let us start from the following formal transformations:

$$
\begin{aligned}
& -i(2 \pi)^{6} \int \frac{d^{4} p}{(2 \pi)^{4}} \frac{k_{1 \mu}}{p^{2}\left(p-k_{1}\right)^{2}(p-q)^{2}}=i \int d^{4} x d^{4} y e^{i\left(k_{1} x+k_{2} y\right)} \frac{\partial}{\partial x^{\mu}}\left(\frac{1}{(x-y)^{2} x^{2} y^{2}}\right) \\
& -i(2 \pi)^{6} \int \frac{d^{4} p}{(2 \pi)^{4}} \frac{k_{2 \mu}}{p^{2}\left(p-k_{1}\right)^{2}(p-q)^{2}}=i \int d^{4} x d^{4} y e^{i\left(k_{1} x+k_{2} y\right)} \frac{\partial}{\partial y^{\mu}}\left(\frac{1}{(x-y)^{2} x^{2} y^{2}}\right)
\end{aligned}
$$

According to the procedure outlined in section (6.3), the l.h.s.'s of these equations will be defined by means of (6.30) and, via Fourier anti-transform, will define the corresponding regularized rational function in the r.h.s.'s. The generalization to multiple powers of the momenta $k_{1}, k_{2}$ in the numerator is straightforward. The (D.1) formulas and the like define a differential regularization.

In the main body of the paper we have to do with similar integrals in which, however, the numerator of the integrand contains polynomials of $p$ beside $k_{1}$ and $k_{2}$. In this case we do not know a straightforward way to differentially regularize them and resort instead to dimensional regularization, in which case other Fourier transforms are needed. For instance

$$
\begin{aligned}
& \int \frac{d^{4} k_{1}}{(2 \pi)^{4}} \frac{d^{4} k_{2}}{(2 \pi)^{4}} \frac{e^{i\left(k_{1}(x-z)+k_{2}(y-z)\right)}}{\left(k_{1}+k_{2}\right)^{2}} \\
& =\frac{1}{16}\left(\int \frac{d^{4} \tilde{k}_{1}}{(2 \pi)^{4}} \frac{e^{i \tilde{k}_{1}\left(\frac{(x-z)+(y-z)}{2}\right)}}{\tilde{k}_{1}^{2}}\right)\left(\int \frac{d^{4} \tilde{k}_{2}}{(2 \pi)^{4}} e^{i \tilde{k}_{2}\left(\frac{x-y}{2}\right)}\right)=\frac{1}{16 \pi^{2}} \frac{1}{(x-z)^{2}} \delta^{(4)}(x-y)
\end{aligned}
$$

where we set $\tilde{k}_{1}=k_{1}+k_{2}$ and $\tilde{k}_{2}=k_{1}-k_{2}$. Proceeding in the same way,

$$
\begin{aligned}
& \int \frac{d^{4} k_{1}}{(2 \pi)^{4}} \frac{d^{4} k_{2}}{(2 \pi)^{4}} \frac{e^{i\left(k_{1}(x-z)+k_{2}(y-z)\right)}}{\left(k_{1}+k_{2}\right)^{2}} \log \left(k_{1}+k_{2}\right)^{2}= \\
& =\frac{1}{4 \pi^{2}} \delta^{(4)}(x-y) \frac{1}{(x-z)^{2}} \log \frac{(x-z)^{2}}{4},
\end{aligned}
$$


and it is understood that

$$
\begin{aligned}
& \int \frac{d^{4} k_{1}}{(2 \pi)^{4}} \frac{d^{4} k_{2}}{(2 \pi)^{4}} e^{i\left(k_{1}(x-z)+k_{2}(y-z)\right.} \log \left(k_{1}+k_{2}\right)^{2}= \\
& =-\left(\partial_{x}+\partial_{y}\right)^{2} \int \frac{d^{4} k_{1}}{(2 \pi)^{4}} \frac{d^{4} k_{2}}{(2 \pi)^{4}} \frac{e^{i\left(k_{1}(x-z)+k_{2}(y-z)\right.}}{\left(k_{1}+k_{2}\right)^{2}} \log \left(k_{1}+k_{2}\right)^{2} .
\end{aligned}
$$

\section{E Conservation of the e.m. tensor}

In this appendix we complete the proof of section 6.4.2.

To start with we write down the structure of the various terms in (6.46) in momentum representation and in coordinate space after applying (1.4)

$$
\begin{aligned}
\epsilon_{\nu \beta \rho \tau} k_{1} \cdot k_{2} k_{1 \lambda} k_{1 \alpha} k_{1}^{\tau} \rightarrow & \int \xi^{\nu} \epsilon_{\nu \beta \rho \tau} \partial_{\sigma} \partial_{\lambda} \partial_{\alpha} \partial^{\tau} h^{\lambda \rho} \partial^{\sigma} h^{\alpha \beta}=0, \\
\epsilon_{\nu \beta \rho \tau} k_{1} \cdot k_{2} k_{1 \lambda} k_{1 \alpha} k_{2}^{\tau} \rightarrow & \int \xi^{\nu} \epsilon_{\nu \beta \rho \tau} \partial_{\sigma} \partial_{\lambda} \partial_{\alpha} h^{\lambda \rho} \partial^{\tau} \partial^{\sigma} h^{\alpha \beta} \\
& =\frac{1}{2} \int \xi^{\nu} \epsilon_{\nu \beta \rho \tau} \partial_{\sigma} \partial^{\rho} \partial_{\alpha} h \partial^{\tau} \partial^{\sigma} h^{\alpha \beta}, \\
\epsilon_{\nu \beta \rho \tau} k_{1} \cdot k_{2} k_{1 \lambda} k_{2 \alpha} k_{2}^{\tau} \rightarrow & \int \xi^{\nu} \epsilon_{\nu \beta \rho \tau} \partial_{\sigma} \partial_{\alpha} h^{\lambda \rho} \partial_{\lambda} \partial^{\tau} \partial^{\sigma} h^{\alpha \beta}, \\
\epsilon_{\nu \beta \rho \tau} k_{1} \cdot k_{2} k_{1 \lambda} k_{2 \alpha} k_{1}^{\tau} \rightarrow & \int \xi^{\nu} \epsilon_{\nu \beta \rho \tau} \partial_{\sigma} \partial_{\alpha} \partial^{\tau} h^{\lambda \rho} \partial_{\lambda} \partial^{\sigma} h^{\alpha \beta}
\end{aligned}
$$

and other similar terms obtained by exchanging 1 and 2. Eq. (E.3) is the opposite of (E.4). In addition we have the term

$$
\eta_{\alpha \lambda} \epsilon_{\nu \beta \rho \tau}\left(k_{1} \cdot k_{2}\right)^{2} k_{1}^{\tau} \rightarrow \int \xi^{\nu} \epsilon_{\nu \beta \rho \tau} \partial_{\sigma} \partial_{\kappa} \partial^{\tau} h^{\lambda \rho} \partial^{\kappa} \partial^{\sigma} h^{\alpha \beta}
$$

and the opposite one obtained by exchanging 1 and 2. All these terms appear with (nonvanishing) coefficients which are rational numbers or rational numbers multiplied by

$$
\frac{2}{\delta}+\gamma-\log 4 \pi+\log 2 k_{1} \cdot k_{2}
$$

in the limit $\delta \rightarrow 0$. The terms proportional to $\log 2 k_{1} \cdot k_{2}$ will be disregarded here because, due to the results in appendix D, they corresponds to the 2-point terms of eq. (1.8). All the other terms have to be canceled by subtracting counterterms from the action. The important point is that such counterterm must be Weyl invariant to the appropriate order in $h$, otherwise they would modify the trace of the e.m. tensor. We show next that this is in fact true for all the above terms.

The terms (E.1) and (E.5) are trivial, for we have

$$
\begin{aligned}
& \delta_{\omega} \int h \epsilon_{\mu \nu \lambda \rho} \partial^{\mu} h^{\tau \nu} \partial^{\lambda} \square h_{\tau}^{\rho}=0, \\
& \delta_{\xi} \int h \epsilon_{\mu \nu \lambda \rho} \partial^{\mu} h^{\tau \nu} \partial^{\lambda} \square h_{\tau}^{\rho}=\int \xi^{\nu} \epsilon_{\nu \sigma \rho \lambda} \partial_{\tau} \partial^{\lambda} \partial^{\kappa} h \partial^{\sigma} \partial_{\kappa} h^{\rho \tau},
\end{aligned}
$$


and

$$
\begin{aligned}
& \delta_{\omega} \int \epsilon_{\mu \nu \lambda \rho} h^{\mu \sigma} \partial^{\tau} \partial^{\lambda} h_{\sigma}^{\rho} \square h_{\tau}^{\nu}=0, \\
& \delta_{\xi} \int \epsilon_{\mu \nu \lambda \rho} h^{\mu \sigma} \partial^{\tau} \partial^{\lambda} h_{\sigma}^{\rho} \square h_{\tau}^{\nu}=-\int \xi^{\nu} \epsilon_{\nu \tau \lambda \rho} \partial^{\tau} \partial^{\kappa} h^{\mu \sigma} \partial_{\mu} \partial_{\kappa} \partial^{\lambda} h_{\sigma}^{\rho}, \\
& \quad+2 \int \xi^{\nu} \epsilon_{\nu \tau \lambda \rho} \partial^{\kappa} \partial^{\alpha} h^{\tau \sigma} \partial_{\kappa} \partial_{\alpha} \partial^{\lambda} h_{\sigma}^{\rho}+\frac{1}{2} \int \xi^{\nu} \epsilon_{\nu \mu \tau \lambda} \partial^{\tau} \partial^{\kappa} h^{\mu \sigma} \partial_{\kappa} \partial^{\lambda} \partial_{\sigma} h .
\end{aligned}
$$

Similarly

$$
\begin{aligned}
\delta_{\omega} \int \epsilon_{\nu \beta \rho \tau} h^{\nu \alpha} \partial_{\kappa} h^{\sigma \rho} \partial_{\sigma} \partial^{\tau} \partial^{\kappa} h_{\alpha}^{\beta}= & 0, \\
\delta_{\xi} \int \epsilon_{\nu \beta \rho \tau} h^{\nu \alpha} \partial_{\kappa} h^{\sigma \rho} \partial_{\sigma} \partial^{\tau} \partial^{\kappa} h_{\alpha}^{\beta}= & \int \xi^{\nu} \epsilon_{\nu \rho \beta \tau} \partial_{\sigma} \partial_{\kappa} h^{\alpha \rho} \partial^{\sigma} \partial^{\kappa} \partial^{\tau} h_{\alpha}^{\beta} \\
& -\frac{1}{2} \int \xi^{\nu} \epsilon_{\nu \rho \beta \tau} \partial^{\rho} \partial_{\kappa} h^{\sigma \tau} \partial_{\sigma} \partial^{\kappa} \partial^{\beta} h,
\end{aligned}
$$

and

$$
\begin{aligned}
& \delta_{\omega} \int \epsilon_{\nu \beta \rho \tau} h^{\nu \sigma} \partial_{\kappa} h^{\alpha \rho} \partial^{\tau} \partial_{\sigma} \partial^{\kappa} h_{\alpha}^{\beta}=0 \\
& \delta_{\xi} \int \epsilon_{\nu \beta \rho \tau} h^{\nu \sigma} \partial_{\kappa} h^{\alpha \rho} \partial^{\tau} \partial_{\sigma} \partial^{\kappa} h_{\alpha}^{\beta}=-\int \xi^{\nu}\left(2 \epsilon_{\nu \beta \rho \tau} \partial_{\kappa} \partial^{\lambda} h^{\alpha \rho} \partial_{\alpha} \partial^{\kappa} \partial^{\tau} h_{\alpha}^{\beta}\right. \\
& \left.+2 \epsilon_{\nu \rho \beta \tau} \partial_{\kappa} \partial^{\rho} h^{\alpha \sigma} \partial_{\sigma} \partial^{\kappa} \partial^{\tau} h_{\alpha}^{\beta}+2 \epsilon_{\nu \beta \rho \tau} \partial^{\kappa} \partial^{\tau} \partial_{\sigma} h_{\alpha}^{\beta} \partial^{\alpha} \partial_{\kappa} h^{\rho \sigma}+\epsilon_{\nu \beta \rho \tau} \partial_{\kappa} \partial^{\rho} h^{\beta \sigma} \partial^{\kappa} \partial^{\tau} \partial_{\sigma} h\right),
\end{aligned}
$$

as well as

$$
\begin{aligned}
& \delta_{\omega} \int \epsilon_{\nu \beta \rho \tau} h^{\nu \sigma} \square h^{\alpha \rho} \partial^{\tau} \partial_{\sigma} h_{\alpha}^{\beta}=0, \\
& \delta_{\xi} \int \epsilon_{\nu \beta \rho \tau} h^{\nu \sigma} \square h^{\alpha \rho} \partial^{\tau} \partial_{\sigma} h_{\alpha}^{\beta}=\int \xi^{\nu}\left(\frac{1}{2} \epsilon_{\nu \beta \rho \tau} \partial_{\kappa} \partial^{\rho} h^{\beta \sigma} \partial_{\sigma} \partial^{\kappa} \partial^{\tau} h\right. \\
& \left.\quad-2 \epsilon_{\nu \rho \beta \tau} \partial_{\kappa} \partial^{\alpha} h^{\rho \sigma} \partial_{\sigma} \partial^{\kappa} \partial^{\tau} h_{\alpha}^{\beta}-\epsilon_{\nu \beta \rho \tau} \partial_{\kappa} \partial^{\rho} h^{\alpha \sigma} \partial^{\kappa} \partial^{\tau} \partial_{\sigma} h_{\alpha}^{\beta}\right),
\end{aligned}
$$

and other similar ones. Using combinations of these relations it is easy to see that all the terms listed above, which appear in (6.46), see (E.1), (E.3) and (E.4), are in fact trivial. They can be reabsorbed in a redefinition of the action without altering the already calculated trace anomaly.

Open Access. This article is distributed under the terms of the Creative Commons Attribution License (CC-BY 4.0), which permits any use, distribution and reproduction in any medium, provided the original author(s) and source are credited.

\section{References}

[1] Y. Nakayama, Scale invariance vs conformal invariance, Phys. Rept. 569 (2015) 1 [arXiv: 1302.0884] [INSPIRE].

[2] S. Rychkov, EPFL Lectures on Conformal Field Theory in $D \geq 3$ Dimensions, https://sites.google.com/site/slavarychkov. 
[3] H. Osborn and A.C. Petkou, Implications of conformal invariance in field theories for general dimensions, Annals Phys. 231 (1994) 311 [hep-th/9307010] [INSPIRE].

[4] J. Erdmenger and H. Osborn, Conserved currents and the energy momentum tensor in conformally invariant theories for general dimensions, Nucl. Phys. B 483 (1997) 431 [hep-th/9605009] [INSPIRE].

[5] S. Weinberg, Six-dimensional Methods for Four-dimensional Conformal Field Theories, Phys. Rev. D 82 (2010) 045031 [arXiv: 1006.3480] [INSPIRE].

[6] M.S. Costa, J. Penedones, D. Poland and S. Rychkov, Spinning conformal correlators, JHEP 11 (2011) 071 [arXiv:1107.3554] [INSPIRE].

[7] Y.S. Stanev, Correlation Functions of Conserved Currents in Four Dimensional Conformal Field Theory, Nucl. Phys. B 865 (2012) 200 [arXiv:1206.5639] [InSPIRE].

[8] A. Zhiboedov, A note on three-point functions of conserved currents, arXiv:1206.6370 [INSPIRE].

[9] Y.S. Stanev, Correlation functions of conserved currents in four dimensional conformal field theory with higher spin symmetry, Bulg. J. Phys. 40 (2013) 147 [arXiv:1312.1467] [INSPIRE].

[10] M.S. Costa and T. Hansen, Conformal correlators of mixed-symmetry tensors, JHEP 02 (2015) 151 [arXiv: 1411.7351] [INSPIRE].

[11] E. Elkhidir, D. Karateev and M. Serone, General three-point functions in 4D CFT, JHEP 01 (2015) 133 [arXiv:1412.1796] [INSPIRE].

[12] A. Cappelli, R. Guida and N. Magnoli, Exact consequences of the trace anomaly in four-dimensions, Nucl. Phys. B 618 (2001) 371 [hep-th/0103237] [INSPIRE].

[13] C. Corianò, L. Delle Rose, E. Mottola and M. Serino, Graviton vertices and the mapping of anomalous correlators to momentum space for a general conformal field theory, JHEP $\mathbf{0 8}$ (2012) 147 [arXiv:1203.1339] [INSPIRE].

[14] A. Bzowski, P. McFadden and K. Skenderis, Implications of conformal invariance in momentum space, JHEP 03 (2014) 111 [arXiv: 1304.7760] [INSPIRE].

[15] D.Z. Freedman, K. Johnson and J.I. Latorre, Differential regularization and renormalization: a new method of calculation in quantum field theory, Nucl. Phys. B 371 (1992) 353 [InSPIRE].

[16] D.Z. Freedman, G. Grignani, K. Johnson and N. Rius, Conformal symmetry and differential regularization of the three gluon vertex, Annals Phys. 218 (1992) 75 [hep-th/9204004] [INSPIRE].

[17] J.I. Latorre, C. Manuel and X. Vilasis-Cardona, Systematic differential renormalization to all orders, Annals Phys. 231 (1994) 149 [hep-th/9303044] [INSPIRE].

[18] H. Osborn, Weyl consistency conditions and a local renormalization group equation for general renormalizable field theories, Nucl. Phys. B 363 (1991) 486 [INSPIRE].

[19] S.M. Christensen and M.J. Duff, Axial and conformal anomalies for arbitrary spin in gravity and supergravity, Phys. Lett. B 76 (1978) 571.

[20] S.M. Christensen and M.J. Duff, New Gravitational Index Theorems and Supertheorems, Nucl. Phys. B 154 (1979) 301 [inSPIRE].

[21] A. Bzowski, P. McFadden and K. Skenderis, Holographic predictions for cosmological 3-point functions, JHEP 03 (2012) 091 [arXiv:1112.1967] [INSPIRE]. 
[22] L. Bonora, S. Giaccari and B. Lima de Souza, Trace anomalies in chiral theories revisited, JHEP 07 (2014) 117 [arXiv: 1403.2606] [INSPIRE].

[23] L. Bonora, P. Pasti and M. Bregola, Weyl cocycles, Class. Quant. Grav. 3 (1986) 635 [INSPIRE].

[24] L. Bonora, P. Pasti and M. Tonin, The anomaly structure of theories with external gravity, J. Math. Phys. 27 (1986) 2259 [INSPIRE].

[25] R.A. Bertlmann, Anomalies in Quantum Field Theory, Oxford Science Publications, Oxford University Press, Oxford U.K. (1996).

[26] K. Fujikawa and H. Suzuki, Path Integrals and Quantum Anomalies, Oxford Science Publications, Oxford University Press, Oxford U.K. (2004).

[27] F. Bastianelli and P. Van Nieuwenhuizen, Path Integrals and Anomalies in Curved Space, Cambridge University Press, Cambridge U.K. (2009).

[28] I.M. Guelfand and G.E. Chilov, Les distributions. Tome 1,2, Dunod, Paris France (1962).

[29] M.J. Duff, Twenty years of the Weyl anomaly, Class. Quant. Grav. 11 (1994) 1387 [hep-th/9308075] [INSPIRE].

[30] R. Delbourgo and A. Salam, The gravitational correction to PCAC, Phys. Lett. B 40 (1972) 381.

[31] A.D. Dolgov and V.I. Zakharov, On conservation of the axial current in massless electrodynamics, Nucl. Phys. B 27 (1971) 525 [inSPIRE]. 